1 Barley RIC157 is involved in RACB-mediated susceptibility to

\title{
2 powdery mildew
}

3 Stefan Engelhardt, Michaela Kopischke, Johanna Hofer, Katja Probst,

4 Christopher McCollum and Ralph Hückelhoven\#

5 Phytopathology, TUM School of Life Science Weihenstephan, Technical University of Munich, Emil-Ramann-Str.2, 85354 Freising, Germany \#Corresponding author: hueckelhoven@wzw.tum.de

\section{Abstract}

Successful pathogens often benefit from certain cellular host processes. For the biotrophic ascomycete fungus Blumeria graminis f.sp. hordei (Bgh) it has been shown that barley RACB, a small monomeric G-protein (ROP, RHO of plants), is required for full susceptibility to fungal penetration. The susceptibility function of RACB probably lies in its role in cell polarisation, which may be co-opted by the pathogen for invasive ingrowth of its haustorium. However, the actual mechnism of how RACB supports the fungal penetration success is little understood. RIC proteins are considered scaffold proteins which can interact directly with ROPs via a conserved CRIB motif. Here we describe a yet uncharacterised RIC protein, RIC157, which can interact directly with RACB. We could show that RIC157 undergoes a recruitment from the cytoplasm to the cell periphery in the presence of activated RACB. During fungal infection, RIC157 and activated RACB colocalise at the penetration site, particularly at the haustorial neck. In a RACB-dependent manner, transiently overexpressed RIC157 renders barley epidermal cells more susceptible to fungal penetration. We conclude that RIC157 promotes fungal penetration into barley epidermal cells via its function as downstream executor in RACB-signaling.

\section{Introduction}

Plants have developed a multilayer immune system to cope with the constant threat of microbial invasion, consisting of pre-formed barriers and induced defences based on the receptor-mediated recognition of microbe-derived and endogenous elicitors (Boller and Felix 2009; Stukenbrock and McDonald 2009). Except for necrotrophs, invading microbes rely to different extents on a living host to establish an infection. With the help 
of secreted effector proteins, pathogens undermine plant immune reactions and influence the host metabolism to render the cellular environment more favourable (Białas et al. 2018, Han and Kahmann 2019). The co-evolution between microbial effectors and their specific host target molecules can lead to an increase in host specialisation, with mutualistic relationships demonstrating extreme examples. Regarding pathogens, especially in genomes of cereal powdery mildew fungi it has been observed that the amount of genes encoding for metabolic enzymes is massively reduced, concomitantly with the proliferation of the putative effector gene pool and transposable elements (Spanu et al. 2010, Wicker et al. 2013, Frantzeskakis et al. 2018). Plant targets of these effectors are not necessarily involved in resistance mechanisms, but also in cellular processes that, when controlled by the pathogen, can become disadvantageous for the host and support the susceptibility towards the invading pathogen. With the current possibilities to use a plethora of different breeding technologies, durable crop resistance based on the loss of these susceptibility gene product functions is within reach (Engelhardt et al. 2018).

Powdery mildew fungi are notorious pathogens known to infect a wide variety of monocot and dicot plants causing massive yield losses in crops. The ascomycete fungus Blumeria graminis f.sp. hordei $(B g h)$ is the specific causal agent of the agronomically important powdery mildew disease on barley (Hordeum vulgare) in Europe (Jørgensen and Wolfe 1994). As an obligate biotrophic parasite, Bgh requires living epidermal cells to complete its life cycle (Hückelhoven and Panstruga 2011). Airborne conidia germinate on the leaf surface and form an appressorium to penetrate the cuticle and the cell wall with the help of an immense turgor pressure and the release of cell wall-degrading enzymes (McKeen and Rimmer 1973; Schulze-Lefert and Vogel 2000; Hückelhoven and Panstruga 2011). A successful fungal infection is characterised by the formation of a haustorium inside the host cell which is essential for nutrient uptake and effector protein delivery (Hahn and Mendgen 2001, Voegele et al. 2001, Panstruga and Dodds 2009). The haustorium is separated from the host cytosol by the extrahaustorial matrix and surrounded by the extrahaustorial membrane (EHM), which is continous with the plant plasma membrane, but differs functionally and biochemically from it (Koh et al. 2005). It is feasible to imagine a pathogen-triggered active contribution of the plant to accomodate the fungal haustorium.

ROPs (RHO (RAS homologue) of plants, or RACs, for rat sarcoma (RAS)-related C3 botulinum toxin substrate) form a unique subfamily of small monomeric RHO GTPases 
in plants, since they do not fall into the phylogenetic RHO subclades of RAC, CDC42 and RHO GTPases found in yeast or animals (Brembu et al. 2006). G-proteins are paradigms of molecular switches due to their ability to bind and hydrolyze GTP. The GTP-bound form represents the activated state, and a plasma membrane association of ROP-GTP via posttranslational lipid modifications is required for downstream signalling (Yalovsky 2015). Upon GTP hydrolysis, GDP-bound or nucleotide-free ROPs are inactive. The cycling between activated and inactive state needs to be spatiotemporally controlled by regulatory partners. Guanine nucleotide exchange factors (GEFs) positively regulate ROP activity by facilitating the GDP/GTP exchange. In plants, three different sorts of ROP GEFs can be distinguished based on their particular GEF domain: PRONE (plant-specific Rop nucleotide exchanger), DHR2 (DOCK homology region 2, found in SPIKE1) and a less well characterized DH-PH domain (B-cell lymphoma homology-pleckstrin homology) described in a plant homolog of human SWAP70 (Berken et al. 2005, Meller et al. 2005; Gu et al. 2006, Basu et al. 2008, Yamaguchi and Kawasaki 2012, Yamaguchi et al. 2012, He et al. 2018). The interaction of ROPs with a GTPase Activating Protein (GAP) enhances the intrinsic GTP hydrolysis activity, followed by ROP inactivation (Berken and Wittinghofer 2008). Beside their putative involvement in ROP recycling, Guanine nucleotide Dissociation Inhibitors (GDIs) bind and sequester inactive ROPs in the cytoplasm and are therefore considered negative regulators of ROP activity (Klahre et al. 2006, Boulter and Garcia-Mata 2010). ROPs are involved in the regulation of a multitude of cellular processes. For instance, the cytoskeleton organisation and consequentially cell shape and function is subject to RHO-like GTPase control (Chen and Friml 2014). In Arabidopsis thaliana xylem vessels, AtROP11 signaling promotes cell wall apposition and shapes cell wall pit boundaries (Sugiyama et al. 2019). Different ROPs are involved in polar cell growth and even function antagonistically during the generation of Arabidopsis thaliana pavement cells (Craddock et al. 2012). Beside cell polarisation and cytoskeleton organisation, ROPs have been also implicated in membrane trafficking and auxin signaling (Yalovsky et al. 2008, Wu et al. 2011). OsRac1 from rice (Oryza sativa), a great example for demonstrating the versatility of ROPs, enhances cell division by regulating OsMAPK6, thereby promoting rice grain yield (Zhang et al. 2019). It has also been shown to regulate immune-related processes like ROS production, defense gene expression and cell death. OsRac1 becomes activated by OsRacGEF1 upon receptor-mediated perception of fungal-derived chitin 
by OsCEBiP and OsCERK1 (Akamatsu et al. 2013). Chitin-perception might also lead to the activation of OsRAC1 by OsSWAP70 (Yamaguchi et al. 2012). Downstream signaling by OsRAC1 is also triggered after recogniton of pathogen effector proteins: Plasma membrane-localised Pit, a nucleotide binding-leucine rich repeat resistance (NLR) protein for the rice blast fungus Magnaporte oryzae, associates with DOCK family GEF OSSPK1, thereby likely activating OsRac1 (Kawano et al. 2010, Kawano et al. 2014, Wang et al. 2018). A recent report regarding an involvement in defence reactions against rice blast mediated by the NLR protein PID3 (Zhou et al. 2019) opens up the possibility of OsRac1 being a downstream hub of other rice NLR proteins. In the barley-powdery mildew interaction, several barley proteins involved in ROP signaling or ROP activity regulation have been shown to influence fungal penetration success. The barley ROP RACB has been shown to act as susceptibility factor (Schultheiss et al. 2002, Schultheiss et al. 2003, Hoefle et al. 2011). In the absence of the pathogen, RACB appears to be involved in cell polarization processes, as stable RACB silencing affects stomatal subsidiary cell and root hair development (Scheler et al. 2016). The expression of a constitutively activated GTP-bound RACB increased fungal penetration efficiency into barley epidermal cells, whereas silencing RACB by RNA interference (RNAi) renders epidermal cells less susceptible against fungal invasion. Two RACB-interacting proteins have been described as negative regulators of RACB function in susceptibility. First, the Microtubule-Associated ROP-GAP1 (MAGAP1) is recruited to the cell periphery by activated RACB, where it limits susceptibility to powdery mildew likely by enhancing the GTP-hydrolizing activity of RACB (Hoefle et al. 2011). Second, activated RACB recruits the cytoplasmic ROP binding kinase1 (RBK1) to the cell periphery and enhances its kinase activity in vitro (Huesmann et al. 2012). Transient silencing of RBK1 or RBK1-interacting protein SKP1 (type II S-phase kinase1-associated protein) suggested that RBK1 acts in negative regulation of RACB protein stability and hence in disease resistance (Reiner et al. 2016). Additionally, RBK1 also appears to be involved in cytoskeleton regulation by positively controling microtubule stability.

In order to regulate cellular processes, ROPs need to activate or deactivate downstream executors (also called ROP effectors). The interaction with some of these executors is often indirect and achieved via scaffold proteins bridging the activated ROPs to their signal destination targets. Two different classes of scaffold proteins have been described so far, ICR/RIPs and RICs. ICR/RIPs (Interactor of Constitutive Active 
ROP/ROP Interactive Partners) have been shown as ROP interactors to be required for cell polarity, vesicle trafficking and polar auxin transport (Lavy et al. 2007, Hazak et al. 2014). In barley, RIPb interacts directly with RACB and enhances disease susceptibility towards powdery mildew (McCollum et al. 2019 Preprint). RIC (ROPInteractive and CRIB-domain containing) proteins, another class of scaffold proteins in ROP signaling, share a highly conserved CRIB motif (Cdc42/Rac Interactive Binding motif, Burbelo et al. 1995), which is essential for the direct interaction with ROPs. The CRIB domain is also present in a subset of ROP GAPs such as barley MAGAP1 (Schaefer et al. 2011). In barley, the knowledge about RIC protein function is quite limited. RIC171, however, has been shown to not only interact directly with RACB, but also to increase fungal penetration efficiency in barley epidermal cells upon overexpression. Activated RACB recruits RIC171 to the cell periphery and, in the presence of Bgh, RIC171 accumulated at the haustorial neck close to the penetration site (Schultheiss et al. 2008). In Arabidopsis thaliana (At), 11 different RIC proteins have been identified, that do not share any clear sequence homology outside their CRIB domain (Wu et al. 2001). By directly interacting with AtROPs, AtRIC proteins are involved in numerous cellular processes. During salt stress, AtROP2 regulates microtubule organisation in an AtRIC1-dependent manner (Li et al. 2017). AtRIC1 also interacts with AtROP6 in pavement cells to enhance the ordering of cortical microtubules upon hormonal signals (Fu et al. 2009) and is involved in cell elongation during pavement cell morphogenesis (Higaki et al. 2017). AtRICs counteract each other to a certain extent as well, as seen with AtROP1-interacting AtRIC3 and AtRIC4 during pollen tube growth. AtRIC3 regulates calcium influx and triggers actin depolymerisation, whereas AtRIC4 enhances actin polymerisation (Gu et al. 2005). Light-induced stomatal opening is regulated via the AtROP2-AtRIC7 pathway. AtROP2 and AtRIC7 are likely to impinge on vesicular trafficking by inhibiting AtExo70B1 which results in a diminished stomatal opening (Hong et al. 2016). These examples emphasize the importance of ROP proteins as signaling hubs for various developmental processes as well as the role RIC proteins play in finetuning the specific cellular responses. However, the function of RICs during the interaction with pathogens still remains to be elucidated.

Here we show results on barley RIC157, another CRIB domain-containing protein that interacts CRIB-dependently with RACB in yeast and in planta. Overexpression of RIC157 increases the powdery mildew penetration efficiency in barley leaf epidermal 
cells in a RACB-dependent manner. Cytosolic RIC157 is recruited to the cell periphery specifically by activated RACB and both proteins co-localise at the haustorial neck during the compatible interaction with Bgh. Our findings indicate a possible role of the RACB-RIC157 signaling pathway in promoting fungal penetration, thereby increasing susceptibility towards Bgh.

\section{Results}

\section{Identification of RIC proteins in barley}

RIC proteins are characterised by their low molecular weight and a lack of sequence homology to any proteins in the database outside their CRIB domain (Wu et al. 2001). This conserved motif has been shown to interact directly with activated small GTPases (Burbelo et al. 1995, Aspenström 1999). In order to identify additional RIC proteins in barley, we performed a BLAST search using the CRIB motif of previously described RACB interacting protein RIC171 (Schultheiss et al. 2008) against the 2019 annotation of all barley coding sequences (Barley all CDS Morex v2.0 2019, https://webblast.ipkgatersleben.de/barley ibsc/). Beside RIC171, we identified another seven proteins sharing the properties of RIC proteins, and named them according to their predicted amino acid sequence length RIC153 (HORVU.MOREX.r2.3HG0258770), RIC157 (HORVU.MOREX.r2.6HG0469110), RIC163 (HORVU.MOREX.r2.5HG0443720), RIC168

(HORVU.MOREX.r2.2HG0170820),

RIC170 (HORVU.MOREX.r2.6HG0521090), RIC171 (HORVU.MOREX.r2.2HG0164690), RIC194 (HORVU.MOREX.r2.3HG0258620), RIC236 (HORVU.MOREX.r2.2HG0122110). An amino acid sequence alignment of all eight barley RIC proteins illustrated no general domain homologies beyond the highly conserved CRIB motif (Fig. 1). Interestingly, the CRIB motif was more C-terminally located in RICs 153, 163 and 194, similar to RIC2 and RIC4 of Arabidopsis thaliana (Wu et al. 2001), while the other RICs (157, 168, 170, 171 and 236) contained the CRIB motif closer to their N-terminal end. However, we didn't identify additional conserved domains shared by all members of the barley RIC protein family except for the CRIB domain.

RACB-mediated susceptibility towards powdery mildew is determined exclusively in barley leaf epidermal cells. Hence, in order to unravel downstream signaling components of RACB, we focused on barley leaf-expressed RIC proteins. Using online available expression databases

(https://webblast.ipk- 
gatersleben.de/barley $\mathrm{ibsc/}$ ), we identified five leaf-expressed $R / C$ genes (RIC153, RIC157, RIC163, RIC194 and previously published RIC171 (Schultheiss et al. 2008). Despite there is little sequence conservation between RIC proteins outside their CRIB domain, we compared primary sequences of barley, Arabidopsis thaliana and rice (Oryza sativa) RICs using an available online amino acid motif discovery software (http://meme-suite.org/tools/meme). Interestingly, this discovered three more amino acid motifs shared by indidual barley (RIC157, RIC168, RIC171), rice (Os02g06660.1, Os04g53580.1) and Arabidopsis RICs (RIC10, RIC11) (Suppl. Fig. S1).

\section{RIC157 increases suspeptibility of barley towards Bgh in a RACB-dependent} manner

To investigate a potential biological function of RIC157 during the barley-powdery mildew interaction, we analysed the penetration success of Bgh on barley epidermal cells during various conditions (Fig. 2). Single cell transient overexpression of RIC157 had a strong effect on the penetration success of Bgh into barley epidermal cells (Fig. 2A). The susceptibility increased by about $50 \%$ compared to control treatments, an outcome that is reminiscent of susceptibility levels observed after transient RACB or RIC171 overexpression (Schultheiss et al. 2003, 2008). Interestingly, we didn't observe the opposite effect, meaning a decreased fungal penetration after RNA interference (RNAi)-mediated silencing of RIC157 compared to control levels (Fig. 2B). This suggests additional factors being involved in RACB-mediated susceptibility towards Bgh. To check if this elevated susceptibility of barley epidermal cells after overexpression of RIC157 is dependent on endogenously present RACB, we analysed the fungal penetration efficiency by simultaneously overexpressing RIC157 and transiently silencing endogenous RACB expression via RNAi (Fig. 2c). Interestingly, we did not observe an elevated fungal success rate, indicating that RIC157 increases barley epidermal cell susceptibility in a RACB-dependent manner. We confirmed the efficiency of both RNAi silencing constructs via co-expression of fluorescence taglabelled targets and ratiometric fluorescence measurements (Suppl. Fig. S2).

\section{RIC157 interacts directly with RACB in yeast and in planta}


approaches to test for direct protein-protein interaction between RIC157 and RACB. In a targeted yeast-2-hybrid experiment, we showed that RIC157 directly interacts with RACB and we also confirmed the CRIB motif being essential for this interaction (Fig. 3A, Suppl. Fig. S3). In yeast, RIC157 directly interacts with RACB and with the constitutively activated RACB mutant CARACB(G15V), but not with the dominantnegative, GDP-bound RACB mutant DNRACB(T20N). Interestingly, we also observed a certain level of interaction between RIC157 and lower nucleotide affinity RACB mutant DNRACB(D121N). This particular mutation has been shown previously in RAS mutant D119N to behave either in a constitutively activated or a dominant-negative way, depending on the experimental setup (Cool et al. 1999). To substantiate these results, we aimed to prove the fusion protein stability by immunoblotting (Suppl. Fig. S4). While all RACB variants were stably expressed and detectable, we were unable to confirm the stability of RIC157 variants in yeast in most of several independent experiments. Together with the fact that the yeast growth on selective medium was quite slow but dependent on the RIC157 construct, this suggests a high RIC157 turnover in yeast. In order to investigate the in planta interaction between RACB and RIC157, we performed Bimolecular Fluorescence Complementation (BiFC, synonym split-Yellow Fluorescent Protein (YFP)) experiments (Walter et al. 2004). We therefore fused Nterminally $\mathrm{N}$ - and C-terminal YFP truncations to RIC157 and RACB variants and transiently co-expressed complementary split-YFP fusion proteins in barley epidermal cells via particle bombardment. YFP fluorescence reconstitution was ratiometrically quantified against a co-expressed cytosolic mCherry fluorescence marker. As shown in Fig. 3C (and Suppl. Fig. S5A), YFP fluorescence was reconstituted to a significantly higher extent when split-YFP fusions of RIC157 were co-expressed with split-YFP fusions of RACB and $\operatorname{CARACB}(\mathrm{G} 15 \mathrm{~V})$, compared to co-expressions with DNRACB(T20N) and DNRACB(D121N). This suggests that in planta, RIC157 might preferantially interact with activated RACB. We confirmed the stability of split-YFP fusion proteins via immunoblot analysis of total protein samples extracted from transformed barley mesophyll protoplasts (Suppl. Fig. S5B). interaction, we further analysed the interaction between RACB and RIC157 by FLIMFRET and in particular the Green Fluorescent Protein (GFP) lifetime reduction (Fig. 3B). We fused mCherry N-terminally to RIC157 and GFP N-terminally to the activated 
CARACB(G15V) and dominant-negative DNRACB(D121N) form and transiently coexpressed mCherry and GFP fusion combinations in barley epidermal cells via particle bombardment. Co-expression of GFP-DNRACB(D121N) and mCherry-RIC157 resulted in an average GFP lifetime of about $2.6 \mathrm{~ns}$, which is very similar to the value we measure for GFP donor only expression. In contrast to that, the co-expression of mCherry-RIC157 with GFP-CARACB(G15V) led to a small but highly significant reduction in GFP lifetime to approximately $2.5 \mathrm{~ns}$. This GFP lifetime reduction clearly demonstrates a direct protein-protein interaction between RIC157 and activated RACB in planta.

\section{Recruitment of RIC157 to the cell periphery is RACB dependent}

Split-YFP fluorescence and GFP fluorescence during FLIM measurements localised predominantly to the cell periphery. To investigate the subcellular localisation of RIC157, we fused GFP N-terminally to RIC157 and transiently overexpressed this construct in barley epidermal cells via biolistic transformation. As shown in Figure 4A (upper row), RIC157 localises to the cytosol, but a lack of GFP fluorescence in the nucleoplasm indicated that GFP-RIC157 is excluded from the nucleus. However, we repeatedly observed a strong fluorescent signal around the nucleus suggesting a potential affinity of RIC157 to the nuclear envelope/endoplasmic reticulum membrane or proteins associated to it.

Since we found a direct protein-protein interaction between RIC157 and RACB, we checked the potential impact of RACB on the subcellular localisation of RIC157 by transiently co-expressing GFP-RIC157 with various untagged RACB forms (Suppl. Fig. S6). The cytoplasmic localisation of RIC157 is not significantly affected in the presence of RACB or the dominant negative DNRACB(D121N) mutant. However, we observed a decrease in cytoplasmic and nuclear envelope-localised GFP fluorescence and an accumulation of GFP fluorescence at the cell periphery when GFP-RIC157 was coexpressed with the constitutively activated CARACB(G15V) form.

Because untagged proteins cannot be properly monitored, we extended our analysis in barley epidermal cells using mCherry-tagged variants of RACB (Fig. 4A). Similarly, we detected GFP-RIC157 in the cytoplasm where it co-localised with mCherry fusions of RACB and DNRACB(D121N). In contrast to that, as with the untagged activated RACB, we detected a similarly strong re-localisation of GFP-RIC157 to the cell periphery in the presence of mCherry-tagged CARACB(G15V) that likewise 
accumulated at this site. This suggests that activated RACB, like other ROPs associating with the plasma membrane probably via its C-terminal prenylation and possible palmitoylation (Schultheiss et al. 2003, Yalovsky 2015), recruits RIC157 to the cell periphery.

In order to check if this RIC157 recruitment to the cell periphery is due to the coexpression with activated RACB, we simultaneously transformed barley epidermal cells with a RNAi construct to silence RACB (Fig. 4B). Without RACB silencing, coexpression of GFP-RIC157 and mCherry-CARACB(G15V) again lead to accumulation of both fusions proteins at the cell periphery. RNAi-mediated silencing of RACB on the other hand did not only diminish the mCherry fluorescence to almost non-detectable levels demonstrating a successful RACB silencing, it also decreased GFP fluorescence at the cell periphery and increases GFP fluorescence in the cytoplasm. This result clearly supports that the observed recruitment of RIC157 to the cell periphery is mediated by activated RACB.

\section{RIC157 and RACB co-localise and accumulate at penetration site}

Co-localisation experiments in unchallenged barley epidermal cells demonstrated a recruitment of RIC157 to the cell periphery in the presence of activated RACB. In order to investigate, if both proteins co-localise at a more specific subcellular site during the interaction with the powdery mildew fungus, we analysed the localisation of transiently co-expressed RIC157 and CARACB(G15V) in epidermal barley cells 18-24 hours after inoculation with Bgh. As shown in Fig. 5, fluorescent protein fusions of RIC157 and activated RACB accumulate close to the fungal penetration site, forming a cone outlining the neck of a developing haustorial initial. The RIC157/CARACB(G15V) colocalisation was much more defined than the fluorescence signal of simultaneously expressed cytoplasmic mCherry (Fig. 5B), indicating a specific membrane-associated co-localisation in epidermal cells that are successfully penetrated by the fungus.

\section{Discussion}

The activity of the ROP protein RACB appears to be disadvantageous for barley in the presence of Bgh. To date, however, our knowledge about the exact RACB-regulated cellular process(es), the fungus takes advantage of, is still quite limited. Albeit we observed a role of RACB or RACB-interacting proteins in polar cell development and 
cytoskeleton organization (Opalski et al. 2005; Hoefle et al. 2011; Huesmann et al. 2012; Scheler et al. 2016; Nottensteiner et al. 2018), a direct mechanistic link between RACB-mediated susceptibility and RACB-regulated cytoskeleton organisation or polar membrane trafficking is still missing. Our studies, however, open up the prospect of a RACB-regulated pathway via a ROP-specific scaffold protein that might be exploited in barley epidermal cells by Bgh to support susceptibility towards powdery mildew.

\section{RIC proteins as scaffolds in RACB downstream signalling}

In a signalling cascade, scaffold proteins are as vital for mediating the molecular response as upstream signalling hubs or downstream executors. These scaffolds establish not just a hub-executor connection, but by doing so they represent also the first branching point in the signalling cascade that eventually leads to specific effects without possessing any kind of enzymatic activity themselves (Zeke et al. 2009, Good et al. 2011). Regarding signalling pathways, ROPs function as signalling hubs and have been shown to be involved in loads of cellular processes and for some ROPs the regulatory role in different, sometimes even antagonistic signalling pathways has been described (Gu et al. 2005; Nibau et al. 2006; Feiguelman et al. 2018). If ROPs do not interact directly with downstream executors, RIC proteins (and also ICRs/RIPs, Mucha et al. 2010, McCollum et al. 2019 Preprint) are considered bridging units creating the scaffold for specific branches of ROP signalling (Schultheiss et al. 2008, Craddock et al. 2012, Zhou et al. 2015, Hong et al. 2016). The presence of a CRIB motif without any further sequence homology to known proteins defines a RIC protein (Wu et al. 2001). The CRIB motif enables RIC proteins to directly interact with ROPs, as for instance described for the Arabidopsis thaliana ROP2-RIC4 and ROP6-RIC1 interaction or for the barley RACB-RIC171 interaction (Fu et al. 2005, Schultheiss et al. 2008, Fu et al. 2009). Beside RIC171, we identified another seven proteins of different sizes in barley that, by the above-mentioned definition, we consider RIC proteins. Apparently there is a difference between monocots and dicots regarding the number of RIC proteins (Wu et al. 2001), similar to RIP proteins (McCollum et al. 2019 Preprint), suggesting a higher probability of either functional redundancies, diversification or antagonistic partners in dicots ( $\mathrm{Gu}$ et al. 2005). Redundancies between the barley leaf-expressed RIC proteins are, considering the low primary sequence conservation, hard to predict and not known yet. The partial similarities between leaf-expressed RIC157 and RIC171 (Suppl. Fig. 1) could however indicate 
that these two RIC proteins are either essential co-ligands, redundant scaffold proteins or even antagonizing members of the same signalling pathway.

\section{RIC157 increases susceptibility towards powdery mildew}

In this study, we concentrated on leaf-expressed RIC157 and show, that the transient RIC157 overexpression leads to a strong increase in barley epidermal cell susceptibility towards powdery mildew infection (Fig. 2A). Thus, the fungus explicitly benefits from a highly abundant RIC157. The RNA interference-mediated silencing of RIC157, however, did not lead to a higher resistance compared to control-treated cells (Fig. 2B). This could have different reasons. Although we have shown a significant reduction of RIC157 protein levels in the presence of the RIC157 RNAi silencing construct (Suppl. Fig. S2), it must be noted that RNAi-based silencing is never $100 \%$ efficient. Remaining endogenous RIC157 transcript levels might be sufficient to allow for control level penetration efficiencies. Additionally, the protein turnover rate of RIC157 is unknown, meaning even with a high RNAi silencing efficiency it is still possible that RIC157 protein, expressed before the transformation with the RNAi silencing construct, is present throughout the infection assay and sufficiently abundant to mask an otherwise more resistant phenotype. Another very important point that needs to be stressed is the function of RACB or ROPs in general as signalling hubs (Nibau et al. 2006). The signalling via RIC157 is likely not the only RACB-regulated path that leads to susceptibility. Shutting down the particular RACB-RIC157 route probably still leaves other RACB signalling pathways functional, which are also involved to a certain extent in RACB-mediated susceptibility. An increased resistance towards fungal infection is only achieved once the signaling hub is removed or switched off by silencing RACB or by transient overexpression of MAGAP1 (Hoefle et al. 2011). In accordance with this, RACB-dependency of the RIC157-promoted susceptibility (Fig. 2C) suggests that even abundant RIC157 still requires the presence of RACB to function as a susceptibility factor.

\section{RIC157 interacts with RACB and is recruited to the cell periphery}

We have demonstrated that RIC157 can interact directly with RACB in yeast and in planta (Fig. 3). In barley epidermal cells, RIC157 showed interaction with activated RACB, but not with either dominant negative form. The observed interaction in yeast between RIC157 and the dominant-negative form DNRACB(D121N) could be 
explained by the different experimental setups and/or biological backgrounds. Gproteins with this particular mutation have been observed previously to behave either dominant-negative or constitutively activated (Cool et al. 1999). DNRACB(D121N) has an intrinsic lower nucleotide affinity, however in yeast this form might be GTP bound and hence resemble the activated RACB.

The subcellular in planta RACB-RIC157 interaction site seen in BiFC and FLIM-FRET experiments appeared to be at the cell periphery. This seems conclusive, because RIC157 preferentially interacted with activated RACB. Activated ROPs are supposed to be associated with negatively charged phospholipids in plasmamembrane nanodomains, which is additionally promoted via posttranslational prenylation and Sacylation (Yalovsky 2015, Platre et al. 2019). Fluorescence-tagged RIC157 alone did not show any specific localisation in the absence of the fungus, although it seems to be excluded from the nucleus (Fig. 4). In the presence of activated RACB, however, RIC157 undergoes a relocalisation from the cytoplasm to the cell periphery, where the interaction with the activated ROP takes place. It had been previously shown that expression of activated GFP-RACB alone leads to its preferential localisation at the cell periphery with a cytosolic background (Schultheiss et al. 2003). We assume that a potential influence of endogenous levels of RIC157 or activated RACB on the localisation of their overexpressed interaction partner is probably negligible in our experimental setup. A similar recruitment to the cell periphery has been observed with other proteins that directly interact with activated RACB (Schultheiss et al. 2008, Huesmann et al. 2012, McCollum et al. 2019 Preprint), reinforcing the model that RACB activation is preceding the recruitment of interaction partners and that downstream RACB signaling is initiated at the plasma membrane. This is further supported since a RACB mutant version lacking the C-terminal CSIL motif for prenylation localizes to the cytoplasm and is inactive in promoting susceptibility (Schultheiss et al. 2003).

With regard to RACB's and RIC157's capability to support fungal infection, the recruitment of RIC157 to the cell periphery becomes even more interesting. Our data suggest a recruitment of RIC157 to the attempted fungal penetration site (Fig. 5). The cone-like structure surrounding the haustorial neck indicates a subcellular colocalisation with activated RACB. However, the co-localisation of RACB and fungal infection-supporting RACB-interactors is not exclusive to RIC157. RIC171 and RIPb, two proteins also considered scaffolds in RACB signalling, co-localise at the haustorial 
neck (Schultheiss et al. 2008, Hückelhoven and Panstruga 2011; McCollum et al. 2019 Preprint). Future experiments may show, whether subcellular co-concentration of RACB and RACB-interacting proteins indicate a specific lipid composition of the haustorial neck, which then recruits activated ROPs, or a membrane domain of high ROP activity due to local GEF activity, or perhaps indicates an exclusion of ROPs from further lateral diffusion into the EHM, which was suggested to be controlled at the haustorial neck (Koh et al. 2005).

\section{RIC157 and susceptibility}

RIC157 transiently overexpressed in barley epidermal cells localises to the cytoplasm and enhances fungal penetration efficiency in a RACB-dependent manner. Activated RACB, however, associates with the plasma membrane where it likely recruits RIC157 for downstream signalling. Thus, transiently overexpressed and endogenous RIC157 might promote RACB-mediated susceptibility upon recruitment to the cell periphery by endogenously present activated RACB. Only a small fraction of overexpressed RIC157 is possibly recruited to the cell periphery without concomitant co-overexpression of activated RACB. This means that such a minute fluorescence localisation change might prove difficult to be detected, but it needs to be emphasized that we do not assume RIC157 promoting susceptibility to powdery mildew from a solely cytoplasmic site. Indeed, in front of a cytoplasmic background, RIC157 is also visible at the cell periphery without co-expression of $\mathrm{CARACB}(\mathrm{G} 15 \mathrm{~V})$, possibly reflecting partial recruitment by endogenous ROPs (Fig. 4A).

The domain and sequence similarity between barley RIC157 and Arabidopsis thaliana RIC10 and RIC11 (Suppl. Fig. 1) does not necessarily indicate similar functions of both proteins. Beside AtRIC10 and AtRIC11, for which nothing is known to date about their biological function, RIC157 also shares some amino acid motif similarity with AtRIC1, AtRIC3 and AtRIC7 (Suppl. Fig. 7), for which an involvement in cytoskeleton organization has previously been demonstrated. From these three Arabidopsis RIC proteins, AtRIC1 appears to be an interesting candidate from which a RIC157 function could be deduced. AtRIC1 has been shown to interact with ROP6 to activate the p60 subunit of Katanin (KTN1), a microtubule-severing enzyme (Lin et al. 2013), as opposed to the interaction with AtROP2 that negatively regulates the action of AtRIC1 on microtubules (Fu et al. 2005). The microtubule-severing activity of KTN1 might even be regulated by AtROP2, AtROP4 and AtROP6 (Ren et al. 2017) via AtRIC1. Whether 
barley RIC157 also fulfils such a regulatory role in organising microtubules still remains to be seen. Regarding the subcellular localisation there are, however, clear differences: Barley RIC157 localises to the cytoplasm, AtRIC1 associates with microtubules (Fu et al. 2005). However, when microtubule arrays in penetrated and attacked but non-penetrated barley epidermal cells were compared, penetration success was strongly associated with parallel non-polarized microtubule arrays in the cell cortex and a diffuse or depleted microtubule structure at the haustorial neck (Hoefle et al. 2011). AtRIC3 has been shown to be involved in the pollen tube growth process, where its function leads to actin disassembly in a ROP1-dependent manner upon calcium influx into the cytoplasm (Gu et al. 2005, Lee et al. 2008). Likewise, AtRIC7 was recently reported to influence vesicle trafficking in stomata resulting in the suppression of an elevated stomatal opening after ROP2-dependent inhibition of the exocyst complex via Exo70B1 (Hong et al. 2016). Both F-actin organization and exocyst function are important in penetration resistance to Bgh (Opalski et al. 2005; Miklis et al. 2007; Ostertag et al. 2012). Therefore, the discovery of RIC157 as a RACBdependent susceptibility factor may pave the way to a better understanding of ROPsteered processes that are pivotal for fungal invasion into barley epidermal cells. The challenge will be to find the downstream factors that RIC157 activates and to understand how RIC157 interacts with RACB in the presence of several other RACB interactors. We assume that several diverse RICs and ICR/RIP proteins could form a cooperative network for orchestrating F-actin, microtubule and membrane organization at the site of fungal entry.

\section{Experimental procedures}

\section{Plant and fungal growth conditions}

Wildtype barley (Hordeum vulgare, cultivar "Golden Promise“) was cultivated in long day conditions (16 hours day light, 8 hours darkness) at a temperature of $18^{\circ} \mathrm{C}$ with a relative humidity of $65 \%$ and a light intensity of $150 \mu \mathrm{mol} \mathrm{s} \mathrm{s}^{-1} \mathrm{~m}^{-2}$.

The biotrophic powdery mildew fungus Blumeria graminis f.sp. hordei A6 was used in all experiments. It was cultivated and propagated on barley "Golden Promise“ under the same condition described above.

\section{Cloning of constructs}


Via a Two-Step Gateway cloning approach, in a first PCR RIC157 (HORVU.MOREX.r2.6HG0469110) was amplified from a barley cDNA pool prepared from leafs and epidermal peels using gene-specific primers RIC157_GW_for and RIC157_GW_rev+STOP (Suppl. Table 1) creating an incomplete Gateway attachment site overhang. A second PCR using primers attB1 and attB2 completed the attachment sites. To create a Gateway entry clone of RIC157, the amplified product was recombined into pDONR223 (Invitrogen) via BP-reaction using Gateway BP Clonase ${ }^{\mathrm{TM}}$ II according to manufacturer's instruction (Thermo Fisher Scientific). To clone $R I C 157 \triangle C R I B$, both fragments upstream and downstream of CRIB motif were amplified seperately using primers RIC157_GW_for and RIC157delCRIB_rev for PCR1, RIC157delCRIB_for and RIC157_GW_rev+STOP for PCR2, creating overlapping overhangs. In PCR3 both fragments together with primers RIC157_GW_for and RIC157_GW_rev+STOP completed RIC157 ACRIB with incomplete Gateway attachment sites overhangs. Completetion of attachment sites and creating an entry clone in pDONR223 was done as described above. To clone RIC157-H37Y-H40Y, a site-directed mutagenesis using primers CRIB157H37\&40Y_for and CRIB157H37\&40Y_rev was performed according to QuikChange $®$ Site-Directed Mutaganesis Protocol (Stratagene). For cloning entry constructs of RACB variants, primers RACB_GW_for and RACB_GW_rev were used to amplify $R A C B$ from previously described constructs (Schultheiss et al. 2003) and cloned into pDONR223 via BP as described abobe. To clone $D N R A C B(D 121 N)$, a sitedirected mutagenesis using primers RACB_D121N_fw and RACB_D121N_rv was performed according to QuikChange ${ }^{\circledR}$ Site-Directed Mutaganesis Protocol (Stratagene).

For RNA interference (RNAi) silencing of RIC157 in barley, we PCR-amplified two RIC157 fragments, a 97bp fragment with primers RIC157_RNAi_Notl_for and RIC157_RNAi_EcoRI_rev containing Notl and EcoRI restriction sites, and a 324bp fragment with primers RIC157_RNAi_EcoRI_for and RIC157_RNAi_Xbal_rev containing EcoRI and Xbal restriction sites. After restriction digest of all sites, ligating into pIPKTA38 via Notl and Xbal sites we created a RIC157_RNAi entry construct lacking the CRIB motif nucleotide sequence to prevent off-target silencing of other CRIB-domain containing RNAs. The RIC157_RNAi sequence was then cloned via LR reaction using Gateway LR Clonase $^{\mathrm{TM}}$ || according to manufacturer's instruction 
541 (Thermo Fisher Scientific) into RNAi expression plasmid pIPKTA30N to create a

542 double-strand RNAi expression construct (Douchkov et al. 2005).

543 For Yeast-2-Hybrid expression clones, entry clones of RIC157 and RACB variants were introduced into prey plasmid pGADT7-GW and pGBKT7-GW via LR reaction using Gateway LR Clonase ${ }^{\mathrm{TM}}$ II according to manufacturer's instruction (Thermo Fisher Scientific). pGADT7-GW and pGBKT7-GW have been modified from pGADT7 and pGBKT7 (Clontech) into a Gateway-compatible form using Gateway ${ }^{\mathrm{TM}}$ Vector Conversion System (Thermo Fisher Scientific). To create RACB variants lacking Cterminal prenylation sequence, a premature STOP-Codon was introduced by sitedirected mutagenesis as described above using primers delCSIL_for and delCSIL_rev. In order to clone BiFC constructs, we PCR amplified a RIC157 full-length fragment with primers RIC157_BamHI_for and RIC157_Kpnl_rev containing BamHI and Kpnl restriction sites. After restriction digest, we ligated this construct into pUCSPYNE(R)173 (Waadt et al. 2008).

555 For localisation and overexpression studies in barley, RIC157 and RACB variants in pDONR223 were used as entry constructs to clone them into various pGY1-based CaMV35S promoter-driven expression vectors (Schweizer et al. 1999) via LR reaction as described above. Empty pGY1 (encoding for no tag) was rendered Gatewaycompatible via Gateway ${ }^{\mathrm{TM}}$ Vector Conversion System (Thermo Fisher Scientific). To create expression vectors for proteins C- or N-terminally tagged by GFP or mCherry, Gateway Reading Frame Cassettes for C- and N-terminal fusions, respectively, were integrated into a pGY1-plasmid backbone upon Xbal digestion and combined at 5' or $3^{3}$ with sequences of monomeric GFP or mCherry. Cloning procedure was performed using In-Fusion HD cloning kit (Takara Bio USA). Constructs for GFP and mCherry upstream or downstream of the Gateway cassette were amplified using primers GW_RfA_mCherry-F, GW_RfA_meGFP-F, GW_RfA_Xba-R, GW_Xba_RfB-F, GW_RfB-R, meGFP-STP-F, mCherry-STP-F, XFP-noSTP_Xba-F, XFP-noSTP-R, meGFP-noSTP-R, mCherry-STP_Xba-R and meGFP-STP_Xba-R.

569 The RACB RNAi construct, RACB BiFC constructs have been described previously 570 (Schultheiss et al. 2003, Schultheiss et al. 2008, Schnepf et al. 2018, McCollum et al. 2019 Preprint). 
574 For transient overexpression in barley, primary leaf epidermal cells of $7 \mathrm{~d}$ old plants were transformed using biolistic bombardment with $1 \mu \mathrm{m}$ gold particles that were coated with $2 \mu \mathrm{g}$ of each test plasmid and additionally with $1 \mu \mathrm{g}$ of a cytosolic transformation marker. After mixing the gold particles with plasmid combinations, $\mathrm{CaCl}_{2}(0.5 \mathrm{M}$ final concentration) and $3.5 \mu \mathrm{l}$ of $2 \mathrm{mg} / \mathrm{ml}$ Protamine (Sigma) were added to each sample. The gold particle solution was incubated at room temperature for $30 \mathrm{~min}$, washed twice with $500 \mu \mathrm{l}$ Ethanol (first $70 \%$, then $100 \%$ ) and eventually dissolved in $6 \mu \mathrm{l} 100 \%$ ethanol per biolistic transformation. After shooting, leaves were incubated at $18^{\circ} \mathrm{C}$.

For localisation and BiFC experiments, leaves were analysed 2 days after transformation. For FRET-FLIM analysis of RACB-RIC157 interaction, barley primary leaves of $7 \mathrm{~d}$ old plants were transiently transformed. Therefore, $2 \mathrm{ug}$ of meGFP-RACB and 1ug mCherry-RIC157 containing plasmids were coated on gold particles for biolistic transformation of single barley epidermal cells.

588 For inoculation with Bgh, fungal spores were manually blown in a closed infection device over transformed leaves either 6 hours after transformation (for microscopic analyses 16 hours after inoculation) or 1 day after transformation (to check penetration efficiency 48 hours after inoculation). To analyse penetration efficiency, a transient assay system based on a cytosolic GUS marker was used as decribed previously (Schweizer et al. 1999). The reporter gene construct pUbiGUSPlus was a gift from Claudia Vickers (Addgene plasmid \# 64402; http://n2t.net/addgene:64402; RRID:Addgene_64402, Vickers et al. 2003). Additionally to overexpression or RNAi silencing constructs, each barley leaf was co-transformed with pUbiGUSPlus. 48 hours after Bgh inoculation, leaves were submerged in GUS staining solution (0.1M Na $2 \mathrm{HPO}_{4} / \mathrm{NaH}_{2} \mathrm{PO}_{4} \mathrm{pH}$ 7.0, 0.01 EDTA, 0.005M Potassium hexacyanoferrat (II), 0.005M Potassium hexacyanoferrat (III), 0.1\% (v/v) Triton X-100, $20 \%$ (v/v) Methanol, 0.5mg/mL 1.5-bromo-4-chloro-3-indoxyl- $\beta$-D-glucuronic acid). For the solution to enter the leaf interior, a vacuum was applied. The leaves were incubated at $37^{\circ} \mathrm{C}$ over night in GUS staining solution and subsequently for at least 24 hours in $70 \%$ Ethanol. Fungal structures were stained with ink-acetate solution (10\% ink, 25\% acetic acid). Transformed cells were identified after GUS staining with light microscopy. An established haustorium was considered a successful penetration and for each sample at least 50 interactions were analysed. Barley epidermal cells transformed with the empty expression plasmid were used as negative control. 


\section{Barley protoplast preparation and transformation}

To prepare protoplasts from barley mesophyll cells, the lower epidermis of primary leaves from 7 day-old barley plants was peeled and the leaves were incubated 3 to 4 hours at room temperature in the darkness while floating with the open mesophyll facing downwards on an enzymatic digestion solution: $0.48 \mathrm{M}$ mannitol, $0.3 \%(\mathrm{w} / \mathrm{v})$ Gamborg B5, 10mM MES pH 5.7, 10mM CaCl2, 0.5\% (w/v) Cellulase R10, 0.5\% (w/v) Driselase, 0.5\% Macerozyme R10. After enzymatic treatment, an equal amount of W5 solution was added: $125 \mathrm{mM} \mathrm{CaCl}_{2}, 154 \mathrm{mM} \mathrm{NaCl}, 5 \mathrm{mM} \mathrm{KCl}, 2 \mathrm{mM} \mathrm{MES}$ pH 5.7. Upon filtering through a $40 \mu \mathrm{m}$ nylon mesh, the protoplasts were pelleted $5 \mathrm{~min}$ at $200 \mathrm{~g}$ and carefully resuspended in $10 \mathrm{ml}$ W5 solution. After another centrifugation step, the protoplast concentration was adjusted to $2 \times 10^{6}$ cells per $\mathrm{mL}$ in MMG solution: $0.4 \mathrm{M}$ mannitol, $15 \mathrm{mM} \mathrm{CaCl} 2,2 \mathrm{mM}$ MES $\mathrm{pH}$ 5.7. For each transformation sample, $1 \mathrm{~mL}$ protoplast solution was mixed with $50 \mu \mathrm{g}$ of each plasmid and $1.1 \mathrm{~mL}$ PEG solution (40\% (w/v) PEG4000, 0.1M mannitol, 0.2M CaCl 2$)$ and incubated 20min at room temperture in the darkness. Afterwards, $4.4 \mathrm{~mL}$ of $\mathrm{W} 5$ solution was added to each transformation and gently mixed. After another pelleting at $200 \mathrm{~g}$, the protoplasts were resuspended in $1 \mathrm{~mL}$ W1 solution (0.5M mannitol, $20 \mathrm{mM} \mathrm{KCL}, 4 \mathrm{mM} \mathrm{MES} \mathrm{pH} \mathrm{5.7)} \mathrm{and}$ incubated in the darkness at room temperature for at least 16 hours.

\section{Yeast-2-Hybrid}

Yeast strain AH109 was transformed with bait (pGBKT7) and prey (pGADT7) constructs by following the small scale yeast transformation protocol from Yeastmaker ${ }^{\mathrm{TM}}$ Yeast Transformation System 2 (Clontech). Upon transformation, yeast cells were plated on Complete Supplement Medium (CSM) plates lacking leucine and tryptophan $(\mathrm{LW})$ and incubated for 3 days at $30^{\circ} \mathrm{C}$. A single colony was taken to inoculate $5 \mathrm{~mL}$ of LW-dropout liquid medium that was incubated with shaking over night at $30^{\circ} \mathrm{C}$. The next day, $2 \mathrm{~mL}$ of culture was pelleted for immunoblot analyses. $7.5 \mu \mathrm{L}$ of undiluted overnight culture (and additionally a 1:10, 1:100 and 1:1000 for control purposes) were dropped on CSM plates lacking leucine and tryptophan, and also on CSM plates lacking leucine, tryptophan and adenine. Plates were incubated for at least 3 days at $30^{\circ} \mathrm{C}$. Growth on CSM-LW plates confirmed the successful transformation of both bait and prey plasmids, while growth on CSM-LWAde plates indicated activation 
of reporter genes. As control for a positive and direct protein-protein interaction we routinely used murine p53 and the SV40 large T-antigen (Li and Fields 1993).

\section{Immunoblot analysis}

For total protein extraction from yeast, we followed the protocol described in Kushnirov (2000) using $2 \mathrm{~mL}$ of over night culture of yeast transformants grown in CSM-LW liquid medium. The extraction of total protein from barley mesophyll protoplasts was performed by pelleting transformed protoplasts $5 \mathrm{~min}$ at $200 \mathrm{~g}$. The pellet was resuspended thoroughly in $50 \mu \mathrm{L}$ of $2 x$ SDS loading buffer by vortexing. A complete protein denaturation was achieved by boiling protoplast samples $10 \mathrm{~min}$ at $95^{\circ} \mathrm{C}$. After shortly spinning down the samples, the stability of fusion proteins in yeast and in planta was assessed via Sodium dodecylsulfate-polyacrylamide gel electrophoresis (SDSPAGE) and immunoblotting on PVDF membranes. Antibodies used for detecting protein bands on PVDF membranes came from SantaCruz Biotechnology (https://www.scbt.com/scbt/cart/cart.jsp): anti-GFP(B-2), anti-cMyc(9E10), anti-HA(F7) and horseradish-peroxidase conjugated anti-mouse. Presence of antibodies on membrane was visually detected by using SuperSignal West Femto Chemiluminescence substrate (ThermoFisher Scientific). Equal protein loading and blotting success was confirmed via Ponceau S-staining of the PVDF membrane.

\section{Microscopy}

Localisation and BiFC experiments were analysed on a Leica TCS SP5 confocal laser scanning microscope. The excitation laser wavelengths were $458 \mathrm{~nm}$ for CFP, $488 \mathrm{~nm}$ for GFP, $514 \mathrm{~nm}$ for YFP and $561 \mathrm{~nm}$ for RFP and mCherry, respectively. The fluorescence emission was collected from 462 to $484 \mathrm{~nm}$ for CFP, from 500-550nm for GFP, from 515 to 550nm for YFP and from 569 to $610 \mathrm{~nm}$ for RFP and mCherry. Barley epidermal cells were imaged via sequential scanning as z-stacks in $2 \mu \mathrm{m}$ increments. Maximum projections of each z-stack were exported as Tiff files from the Leica LAS AF software (version 3.3.0).

Localisation experiments of fluorescent protein fusions and BiFC analysis in barley epidermal cells were conducted from 24 hours until 48 hours after biolistic transformation. Regarding raciometric BiFC quantification using Leica LAS AF software (version 3.3.0), the fluorescence intensity was evaluated over the whole cell area and the ratio between YFP and cytosolic mCherry fluorescence signal was 
675 calculated. For each BiFC combination, the fluorescence of at least 20 cells was 676 measured.

677 For FRET-FLIM analysis of RACB-RIC157 interaction, the expression of the 678 fluorophore-fusion proteins was analysed 2 days after transformation using an 679 Olympus FluoView ${ }^{\mathrm{TM}} 3000$ inverse laser scanning confocal microscope with an 680 UPLSAPO 60XW 60x/NA 1.2/WD 0.28 water immersion objective (Olympus, 681 Hamburg, Germany). Fluorescence of GFP was collected between 500-540nm and 682 mCherry emission was imaged between 580-620nm upon excitation with 488 and $561 \mathrm{~nm}$ argon laser lines, respectively. For FRET-FLIM measurements the PicoQuant advanced FCS/FLIM-FRET/rapidFLIM upgrade kit (PicoQuant, Berlin, Germany) was used, comprising a 485nm pulsed laser line for GFP excitation (pulse rate $40 \mathrm{mHz}$, laser driver: PDL 828 SEPIA II, laser: LDH-D-C-485), a Hybrid Photomultiplier Detector Assembly 40 to detect GFP fluorescence and a TimeHarp 260 PICO Time-Correlated Single Photon Counting module (resolution 25 ps) to measure photon life times. GFP fluorescence was imaged at the aequatorial plane of epidermis cells to capture GFP fluorescence at the cell periphery and possibly plasma membrane. For each interaction at least 16 cells were analysed and 300 to 600 photons per pixel were recorded. Decay data within a region of interest were fitted using an n-exponential reconvolution fit with model parameters $n=3$ and calculated instrument response function of the PicoQuant SymPhoTime 64 software.

\section{Supplemental Data}

697 Suppl. Fig. S1: Barley RIC157 shows limited sequence similarity to RIC1 from Arabidopsis thaliana

Suppl. Fig. S2: RNA interference silencing efficiency

Suppl. Fig. S3: CRIB deletion and CRIB mutation in RIC157 prevents interaction with RACB in yeast 
Suppl. Fig. S7: Barley RIC157 shows limited sequence similarity to RIC proteins from Arabidopsis thaliana.

Suppl. Table 1: Primers used in this study

\section{References}

Akamatsu, A., Wong, H.L., Fujiwara, M., Okuda, J., Nishide, K., Uno, K., Imai, K., OsCEBiP/OsCERK1-OsRacGEF1-OsRac1 module is an essential early component of chitin-induced rice immunity. Cell Host Microbe 13, 465-476.

Aspenström, P. (1999). Effectors for the Rho GTPases. Curr. Opin. Cell Biol. 11, 95102.

Basu, D., Le, J., Zakharova, T., Mallery, E.L. and Szymanski, D.B. (2008). A SPIKE1 signaling complex controls actin-dependent cell morphogenesis through the heteromeric WAVE and ARP2/3 complexes. Proc. Natl. Acad. Sci. USA 105, 40444049.

Berken, A., Thomas, C. and Wittinghofer, A. (2005). A new family of RhoGEFs activates the Rop molecular switch in plants. Nature 436, 1176-1180.

Berken, A. and Wittinghofer, A. (2008). Structure and function of Rho-type molecular switches in plants. Plant Physiol. Biochem. 46, 380-393.

Białas, A., Zess, E.K., De la Concepcion, J.C., Franceschetti, M., Pennington, H.G., Yoshida, K., Upson, J.L., Chanclud, E., Wu, C.-H., Langner, T., Maqbool, A., Varden, F.A., Derevnina, L., Belhaj, K., Fujisaki, K., Saitoh, H., Terauchi, R., Banfield, M.J. and Kamoun, S. (2018). Lessons in effector and NLR biology of plantmicrobe systems. Mol. Plant Microbe Interact. 31, 34-45. 
Boller, T. and Felix, G. (2009). A renaissance of elicitors: perception of microbeassociated molecular patterns and danger signals by pattern-recognition receptors. Annu. Rev. Plant Biol. 60, 379-406.

Boulter, E., and Garcia-Mata, R. (2010). RhoGDI: A rheostat for the Rho switch. Small GTPases 1, 65-68.

Brembu, T., Winge, P., Bones, A.M. and Yang, Z. (2006). A RHOse by any other name: a comparative analysis of animal and plant Rho GTPases. Cell Res. 16, 435445 .

Burbelo, P.D., Drechsel, D. and Hall, A. (1995). A conserved binding motif defines numerous candidate target proteins for both Cdc42 and Rac GTPases. J. Biol. Chem. 270, 29071-29074.

Chen, X. and Friml, J. (2014). Rho-GTPase-regulated vesicle trafficking in plant cell polarity. Biochem. Soc. Trans. 42, 212-218.

Cool, R.H., Schmidt, G., Lenzen, C.U., Prinz, H., Vogt, D. and Wittinghofer, A. (1999). The Ras mutant D119N is both dominant negative and activated. Mol. Cell. Biol. 19, 6297-6305.

Craddock, C., Lavagi, I. and Yang, Z. (2012). New insights into Rho signaling from plant ROP/Rac GTPases. Trends Cell Biol. 22, 492-501.

Douchkov, D., Nowara, D., Zierold, U. and Schweizer, P. (2005). A high-throughput gene-silencing system for the functional assessment of defense-related genes in barley epidermal cells. Mol. Plant Microbe Interact. 18, 755-761. 
Edgar, C. (2004). MUSCLE: multiple sequence alignment with high accuracy and high throughput. Nucleic Acids Res. 32, 1792-1797.

Engelhardt, S., Stam, R. and Hückelhoven, R. (2018). Good riddance? Breaking

Feiguelman, G., Fu, Y. and Yalovsky, S. (2018). ROP GTPases structure-function and signaling pathways. Plant Physiol. 176, 57-79.

Frantzeskakis, L., Kracher, B., Kusch, S., Yoshikawa-Maekawa, M., Bauer, S., Pedersen, C., Spanu, P.D., Maekawa, T., Schulze-Lefert, P. and Panstruga, R. (2018). Signatures of host specialisation and a recent transposable element burst in the dynamic one-speed genome of the fungal barley powdery pathogen. BMC Genomics 19, 381.

Fu, Y., Gu, Y., Zheng, Z., Wasteneys, G. and Yang, Z. (2005). Arabidopsis interdigitating cell growth requires two antagonistic pathways with opposing action on cell morphogenesis. Cell 120, 687-700.

Fu, Y., Xu, T., Zhu, L., Wen, M. and Yang, Z. (2009). A ROP GTPase signaling pathway controls cortical microtubule ordering and cell expansion in Arabidopsis. Curr. Biol. 19, 1827-1832.

Good, M.C., Zalatan, J.G. and Lim, W.A. (2011). Scaffold proteins: Hubs for 
790 Gu, Y., Fu, Y., Dowd, P., Li, S., Vernoud, V., Gilroy, S. and Yang, Z. (2005). A Rho 791 family GTPase controls actin dynamics and tip growth via two counteracting downstream pathways in pollen tubes. J. Cell Biol. 169, 127-138.

793

Gu, Y., Li, S., Lord, E.M. and Yang, Z. (2006). Members of a novel class of Arabidopsis Rho guanine nucleotide exchange factors control Rho GTPase-dependent polar growth. Plant Cell 18, 366-381.

Hahn, M. and Mendgen, K. (2001). Signal and nutrient exchange at biotrophic plantfungus interfaces. Curr. Opin. Plant Biol. 4, 322-327.

800

Han, X. and Kahmann, R. (2019). Manipulation of phytohormone pathways by effectors of filamentous pathogens. Front. Plant Sci. 10, 822. in regulation of auxin distribution. PLoS Biol. 8, e1000282. P.R.J. (2018). Plant pathogen effector utilizes host susceptibility factor NLR1 to degrade the immune regulator SWAP70. Proc. Natl. Acad. Sci. USA 115, E7834E7843.

Higaki, T., Takigawa-Imamura, H., Akita, K., Kutsuna, N., Kobayashi, R., Hasezawa, S. and Miura, T. (2017). Exogenous cellulase switches cell interdigitation to cell elongation in an RIC1-dependent manner in Arabidopsis thaliana cotyledon pavement cells. Plant Cell Physiol. 58, 106-119. 
Hoefle, C., Huesmann, C., Schultheiss, H., Börnke, F., Hensel, G., Kumlehn, J. and Hückelhoven, R. (2011). A barley ROP GTPase ACTIVATING PROTEIN associates with microtubules and regulates entry of the barley powdery mildew fungus into leaf epidermal cells. Plant Cell 23, 2422-2439.

Hong, D., Jeon, B.W., Kim, S.Y., Hwang, J.-U. and Lee, Y. (2016). The ROP2-RIC7 pathway negatively regulates light-induced stomatal opening by inhibiting exocyst subunit Exo70B1 in Arabidopsis. New Phytol. 209, 624-635.

Huesmann, C., Reiner, T., Hoefle, C., Preuss, J., Jurca, M.E., Domoki, M., Feher, A. and Hückelhoven, R. (2012). Barley ROP binding kinase 1 is involved in microtubule organization and in basal penetration resistance to the barley powdery mildew fungus. Plant Physiol. 159, 311-320.

Hückelhoven, R. and Panstruga, R. (2011). Cell biology of the plant-powdery mildew interaction. Curr. Opin. Plant Biol. 14, 738-746.

Jørgensen, J.H. and Wolfe, M. (1994). Genetics of powdery mildew resistance in barley. Crit. Rev. Plant Sci. 13, 97-119.

McKeen, W.E. and Rimmer, S.R. (1973). Initial penetration process in powdery mildew infection of susceptible barley leaves. Phytopathology 63, 1049-1053.

Kawano, Y., Akamatsu, A., Hayashi, K., Housen, Y., Okuda, J., Yao, A., Nakashima, A., Takahashi, H., Yoshida, H., Wong, H.L., Kawasaki, T. and Shimamoto, K. (2010). Activation of a Rac GTPase by the NLR family disease resistance protein Pit plays a critical role in rice innate immunity. Cell Host Microbe 7, 362-375. 
Kawano, Y., Fujiwara, T., Yao, A., Housen, Y., Hayashi, K. and Shimamoto, K.

848 (2014). Palmitoylation-dependent membrane localisation of the rice resistance protein

849 Pit is critical for the activation of the small GTPase OsRac1. J. Biol. Chem. 289, 1907919088

Klahre, U., Becker, C., Schmitt, A.C. and Kost, B. (2006). Nt-RhoGDI2 regulates Rac/Rop signaling and polar cell growth in tobacco pollen tubes. Plant J. 46, 10181031.

Koh, S., Andre, A., Edwards, H., Ehrhardt, D. and Somerville, S. (2005). Arabidopsis thaliana subcellular responses to compatible Erysiphe cichoracearum infections. Plant J. 44, 516-529.

859

Kushnirov, V.V. (2000). Rapid and reliable protein extraction from yeast. Yeast 16, 857-860.

Lavy, M., Bloch, D., Hazak, O., Gutman, I., Poraty, L., Sorek, N., Sternberg, H. and Yalovsky, S. (2007). A novel ROP/RAC effector links cell polarity, root-meristem maintenance, and vesicle trafficking. Curr. Biol. 17, 947-952.

Lee, Y.J., Szumlanski, A., Nielsen, E. and Yang, Z. (2008). Rho-GTPase-dependent filamentous actin dynamics coordinate vesicle targeting and exocytosis during tip growth. J. Cell. Biol. 181, 1155-1168.

Li, B. and Fields, S. (1993). Identification of mutations in p53 that affect its binding to SV40 large T antigen by using the yeast two-hybrid system. FASEB J. 7, 957-963. 
Li, C., Lu, H., Li, W., Yuan, M. and Fu, Y. (2017). A ROP2-RIC1 pathway fine-tunes microtubule reorganization for salt tolerance in Arabidopsis. Plant, Cell Environm. 40, 1127-1142.

Lin, D., Cao, L., Zhou, Z., Zhu, L., Ehrhardt, D., Yang, Z. and Fu, Y. (2013). Rho GTPase signaling activates microtubule severing to promote microtubule ordering in Arabidopsis. Curr. Biol. 23, 290-297.

McCollum, C., Engelhardt, S. and Hückelhoven, R. (2019): ROP INTERACTIVE PARTNER $b$ interacts with the ROP GTPase RACB and supports fungal penetration into barley epidermal cells. bioRxiv 750265 .

Meller, N., Merlot, S. and Guda, C. (2005). CZH proteins: a new family of Rho-GEFs. J. Cell. Sci. 118, 4937-4946.

Miklis, M., Consonni, C., Bhat, R.A., Lipka, V., Schulze-Lefert, P. and Panstruga, R. (2007). Barley MLO modulates actin-dependent and actin-independent antifungal defense pathways at the cell periphery. Plant Physiol. 144, 1132-1143.

Mucha, E., Hoefle, C., Hückelhoven, R. and Berken, A. (2010). RIP3 and AtKinesin$13 \mathrm{~A}$ - a novel interaction linking Rho proteins of plants to microtubules. Eur. J. Cell Biol. 89, 906-916.

Nibau, C., Wu, H.M. and Cheung, A.Y. (2006). RAC/ROP GTPases: ,hubs' for signal integration and diversification in plants. Trends Plant Sci. 11, 309-315.

Nottensteiner, M., Zechmann, B., McCollum, C., Hückelhoven, R. (2018). A Barley powdery mildew fungus non-autonomous retrotransposon encodes a peptide that supports penetration success on barley. J. Exp. Bot. 69, 3745-3758. 
Opalski, K.S., Schultheiss, H., Kogel, K.H. and Hückelhoven, R. (2005). The receptor-like MLO protein and the RAC/ROP family G-protein RACB modulate actin reorganization in barley attacked by the biotrophic powdery mildew fungus Blumeria graminis f.sp. hordei. Plant J. 41, 291-303.

Ostertag, M., Stammler, J., Douchkov, D., Eichmann, R. and Hückelhoven, R. (2013). The conserved oligomeric Golgi complex is involved in penetration resistance of barley to the barley powdery mildew fungus. Mol. Plant Pathol. 14, 230-240.

Panstruga, R. and Dodds, P.N. (2009). Terrific protein traffic: The mystery of effector protein delivery by filamentous plant pathogens. Science $324,748-750$.

Platre, M.P. et al. (2019). Developmental control of plant Rho GTPase nanoorganization by the lipid phosphatidylserine. Science $364,57-62$.

Reiner, T., Hoefle, C. and Hückelhoven, R. (2016). A barley SKP1-like protein controls abundance of the susceptibility factor RACB and influences the interaction of barley with the barley powdery mildew fungus. Mol. Plant Pathol. 17, 184-195.

Ren, H., Dang, X., Cai, X., Yu. P., Li, Y., Zhang, S., Liu, M., Chen, B. and Lin, D. (2017). Spatio-temporal orientation of microtubules controls conical cell shape in Arabidopsis thaliana petals. PLoS Genet. 13, e1006851.

Schaefer, A., Höhner, K., Berken, A. and Wittinghofer, A. (2011). The unique plant RhoGAPs are dimeric and contain a CRIB motif required for affinity and specificity towards cognate small G proteins. Biopolymers 95, 420-433. 

Barley disease susceptibility factor RACB acts in epidermal cell polarity and positioning of the nucleus. J. Exp. Bot. 67, 3263-3275.

Schnepf, V., Vlot, C.A., Kugler, K. and Hückelhoven, R. (2018). Barley susceptibility factor RACB modulates transcript levels of signalling protein genes in compatible interactions with Blumeria graminis f.sp. hordei. Mol. Plant Pathol. 19, 393-404.

Schultheiss, H., Dechert, C., Kogel, K.-H. and Hückelhoven R. (2002). A small GTP-binding host protein is required for entry of powdery mildew fungus into epidermal cells of barley. Plant Physiol. 128, 1447-1454.

Schultheiss, H., Dechert, C., Kogel, K.-H. and Hückelhoven, R. (2003). Functional analysis of barley RAC/ROP G-protein family members in susceptibility to the powdery mildew fungus. Plant J. 36, 589-601.

Schultheiss, H., Preuss, J., Pircher, T., Eichmann, R. and Hückelhoven, R. (2008). Barley RIC171 interacts with RACB in planta and supports entry of the powdery mildew fungus. Cell. Microbiol. 10, 1815-1826.

Schulze-Lefert, P. and Vogel, J. (2000). Closing the ranks to attack by powdery mildew. Trends in Plant Science 5, 343-348.

Schweizer, P., Christoffel, A. and Dudler, R. (1999). Transient expression of members of the germin-like gene family in epidermal cells of wheat confers disease resistance. Plant J. 20, 541-552.

Spanu, P.D. et al. (2010). Genome expansion and gene loss in powdery mildew fungi reveal tradeoffs in extreme parasitism. Science 330, 1543-1546. 
Stukenbrock, E.H., McDonald, B.A. (2009) Population genetics of fungal and oomycete effectors involved in gene-for-gene interactions. Mol Plant Microbe Interact. 22, 371-380.

Sugiyama, Y., Nagashima, Y., Wakazaki, M., Sato, M., Toyooka, K., Fukuda, H. and Oda, Y. (2019). A Rho-actin signaling pathway shapes cell wall boundaries in Arabidopsis xylem vessels. Nat. Commun. 10, 468.

Vickers, C.E., Xue, G.P. and Gresshoff, P.M. (2003). A synthetic xylanase as a novel reporter in plants. Plant Cell Rep. 22, 135-140.

Voegele, R.T., Struck, C., Hahn, M. and Mendgen, K. (2001). The role of haustoria in sugar supply during infection of broad bean by the rust fungus Uromyces fabae. Proc. Natl. Acad. Sci. USA 98, 8133-8138.

Waadt, R., Schmidt, L.K., Lohse, M., Hashimoto, K., Bock, R. and Kudla, J. (2008). Multicolor bimolecular fluorescence complementation reveals simultaneous formation of alternative CBL/CIPK complexes in planta. Plant J. 56, 505-516.

Walter, M., Chaban, C., Schütze, K., Batistic, O., Weckermann, K., Näke, C., (2004). Visualization of protein interactions in living plant cells using bimolecular fluorescence complementation. Plant J. 40, 428-438.

Wang, Q., Li, Y., Ishikawa, K., Kosami, K.I., Uno, K., Nagawa, S., Tan, L., Du, J., OsSPK1 to activate OsRac1 and trigger rice immunity. Proc. Natl. Acad. Sci. USA 115, 
Wicker, T. et al. (2013). The wheat powdery mildew genome shows the unique evolution of an obligate biotroph. Nat. Genet. 45, 1092-1096.

991

Wu, G., Gu, Y., Li, S. and Yang, Z. (2001). A genome-wide analysis of Arabidopsis Rop-interactive CRIB motif-containing proteins that act as ROP GTPase targets. Plant Cell 13, 2841-2856.

Wu, H., Hazak, O., Cheung, A.Y. and Yalovsky, S. (2011). RAC/ROP GTPases and auxin signaling. Plant Cell 23, 1208-1218.

Yalovsky, S. (2015). Protein lipid modifications and the regulation of ROP GTPase function. J. Exp. Bot. 66, 1617-1624.

1001

Yalovsky, S., Bloch, D., Sorek, N. and Kost, B. (2008). Regulation of membrane trafficking, cytoskeleton dynamics, and cell polarity by ROP/RAC GTPases. Plant Physiol. 147, 1527-1543.

Yamaguchi, K. and Kawasaki, T. (2012). Function of Arabidopsis SWAP70 GEF in immune response. Plant Signal Behav. 7, 465-468.

Yamaguchi, K., Imai, K., Akamatsu, A., Mihashi, M., Hayashi, N., Shimamoto, K. and Kawasaki, T. (2012). SWAP70 functions as a Rac/Rop guanine nucleotideexchange factor in rice. Plant J. 70, 389-397.

Zeke, A., Lukacs, M., Lim, W.A. and Remenyi, A. (2009). Scaffolds: interaction platforms for cellular signalling circuits. Trends Cell Biol. 19, 364-374. 
Zhang, Y., Xiong, Y., Liu, R., Xue, H.W. and Yang, Z. (2019). The Rho-family GTPase OsRAC1 controls rice grain size and yield by regulating cell division. Proc. Natl. Acad. Sci. USA 116, 16121-16126.

1019

Zhou, Z., Shi, H., Chen, B., Zhang, R., Huang, S. and Fu, Y. (2015). Arabidopsis RIC1 severs actin filaments at the apex to regulate pollen tube growth. Plant Cell 27,

1022 1140-1161.

1023

Zhou, Z., Pang, Z., Zhao,S., Zhang, L., Lv, Q., Yin, D., Li, D., Xue, L., Zhao, X., Li, X., Wang, W. and Zhu, L. (2019). Importance of OsRAC1 and RAI1 in signalling of nucleotide-binding site leucine-rich repeat protein-mediated resistance to rice blast disease. New Phytol. 223, 828-838. 
bioRxiv preprint doi: https://doi.org/10.1101/848226; this version posted November 20, 2019. The copyright holder for this preprint (which was not certified by peer review) is the author/funder, who has granted bioRxiv a license to display the preprint in perpetuity. It is made available under aCC-BY-NC-ND 4.0 International license.

Figures

HvRIC163
HvRIC153
HvRIC170
HvRIC194
HvRIC236
HvRIC157
HvRIC171
HvRIC168

1 MKDRRAGAGFPFS I GCMSQSAVAVADPLEKKPMPPPPAQQQADTPSSSTTAATTQERSAGEESGEDKARNAAASG - IVSA DDPLEKKPIPPDPAOQQ 80

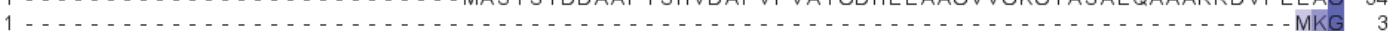
MGSREQRGRRDFIVIPFSSTCRSAASVDIVQSKKPQGAGGGGEGTSAAAVVRPAKG-ESLSL 63

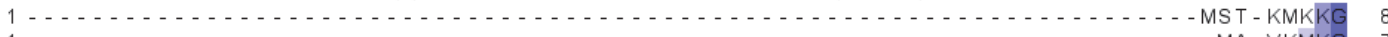
1

\section{| A}

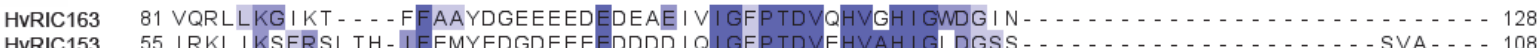
HVRIC153 55 IRKL I KSFRSLTH - I FEMYEDGDEEEEDDDD I I IGFPTDVEHVAH I GLDGSS - . - . - . - .

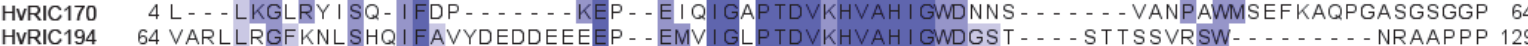
HVRIC194 HvRIC236 HvRIC157 HvRIC171
HvRIC168

9 I - - - LRPFRYFSN - I IDA - -

8 । . . . FKGLR I FSH - MFAA . . . . . QK

$8 \mathrm{~V}$ - . - FKGLRVITQ DST - - STTSSVRSW- UDG HvRIC168

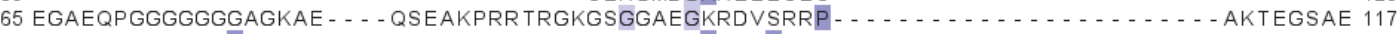

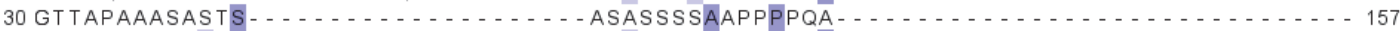
77 DRGGSAASNPWASQE I VLDGAGLGDNSFRDTKSEAGG I EVTAGD SPPSPGTRRSRRNRSRGSDTSSMDVTTGI TDTSEKKE 157 68 STAEQSRQT SWTSTDFEP - . . . . . . . . ARSMLPTEI NFPDRP $\ldots \ldots \ldots$

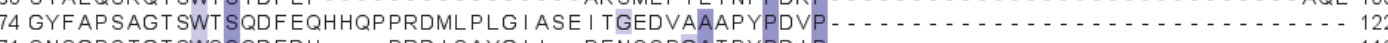
71 SNSGPSTGT SWSSQDFDH - . - PRDI SAYGI I - PENSSPGATPYPDIP . . . . . . . . . . . . . . . . . . 113

141 - - - SSLSLR - - QLE | AMDPGASTTTCIN- - -

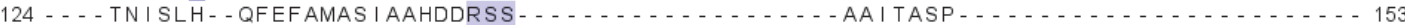
118 AGEGDAAAPKQ-RRRKSKTAGGASGGRSK - . - . - . - SGSGGAASDPEAAKSASAEADDDGR - . . . . . . .

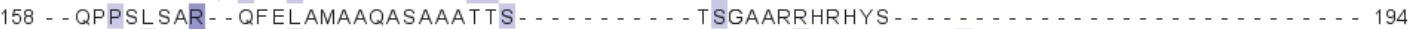
158 KAKKGTRKNR - - KKDKDKATEDTAGSTCQDLPAVPKKSNRRKNKGSSEGSGGASTKDGGGVPEEGTTPLTLVAEEEKDHEL 236 104 SSSCPPRGPRKARRKKTRTSSPTSSARSS . . . . . . . . . S SRSRASFATAFDDF SE SQRGVRVV . . . . . . . . 157

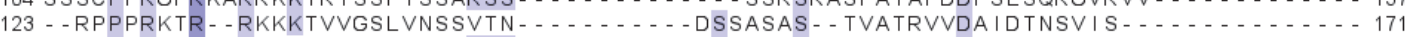

1032 Figure 1: Barley RIC proteins alignment. Multiple alignment

1033 (https://www.ebi.ac.uk/Tools/msa/muscle, Edgar 2004; illustrated using Jalview 2.11.0 software) of predicted barley (Hordeum vulgare, $H v$ ) proteins harboring CRIB domain 1035 (underlined). Intensity of blue coloured amino acids represents level of conservation 1036 (higher intensity = more conserved). CRIB domain consensus sequence of barley RIC proteins is indicated by coloured amino acid sequence above the alignment (http://meme-suite.org/tools/meme). 

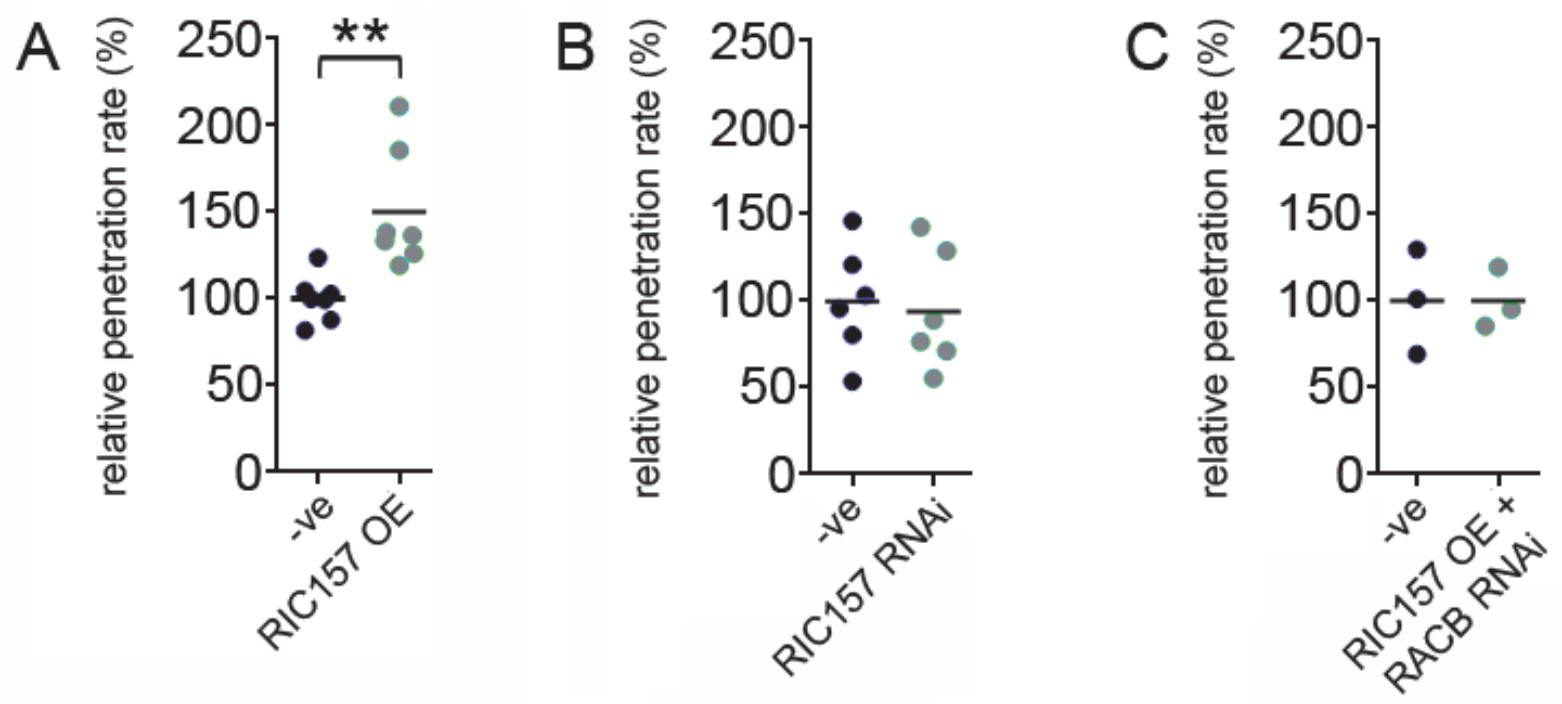

Figure 2: RIC157 increases susceptibility in a RACB-dependent manner.

1042 Epidermal cells of 7 day old primary barley leaves were transiently transformed by 1043 particle bombardment with either (A) an overexpression construct of RIC157, (B) a 1044 RNA interference construct of RIC157 or (C) simultaneously with an overexpression construct of RIC157 and a RNA interference construct of RACB. Empty overexpression or RNA interference plasmids were used as controls (-ve). The penetration efficiency of Bgh into transformed barley epidermal cells was analysed 48 after inoculation with fungal spores. Values are shown as mean of at least 3 independent biological replicates, relative to the mean of the control set as $100 \%$. ${ }^{* *}$ indicates significance $\mathrm{P}$ $<0.01$, Student's t-test. 

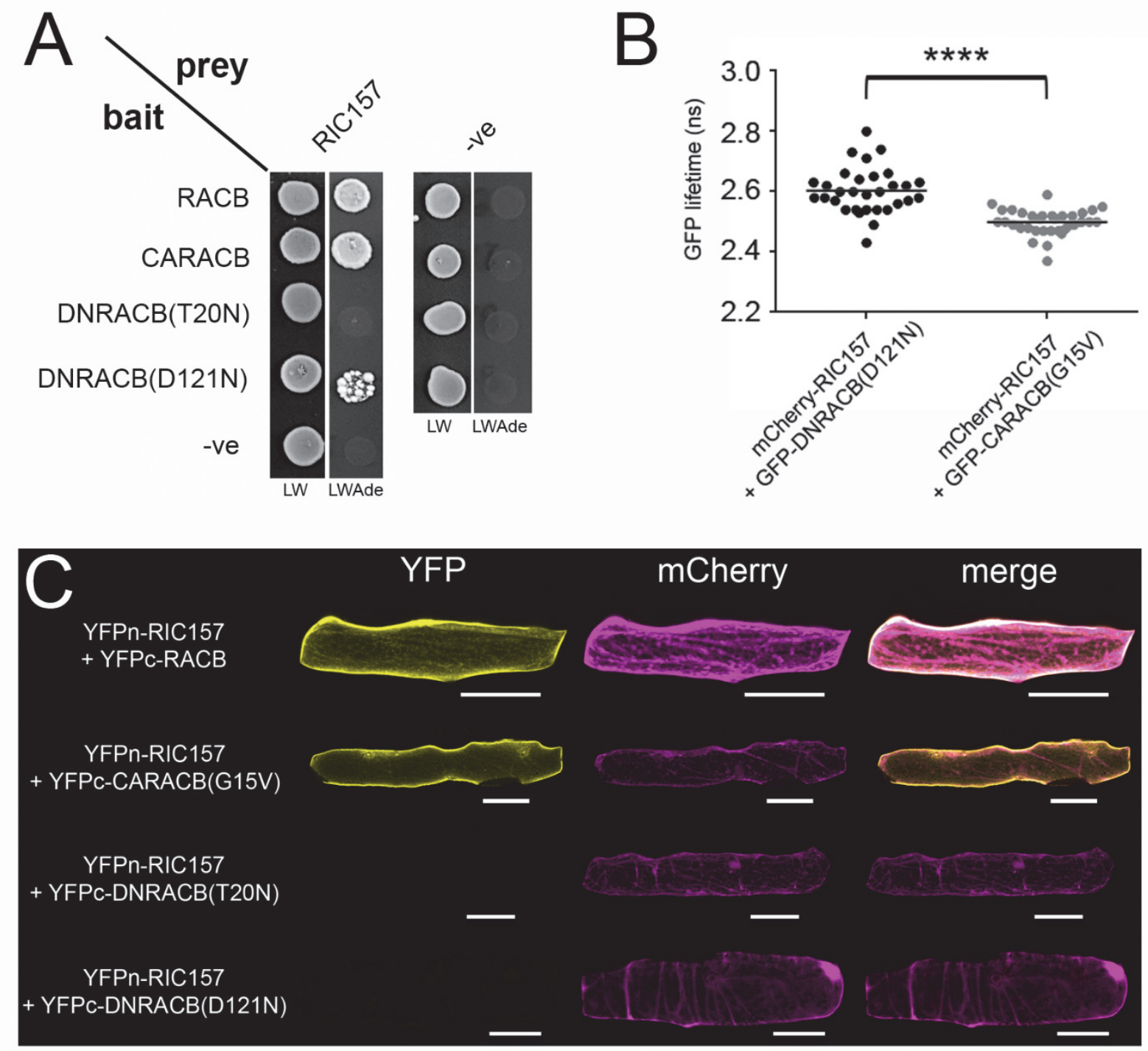

Figure 3: RIC157 interacts directly with RACB in yeast and in planta. A) Yeast-2-

Hybrid indicates direct interaction between RIC157 and RACB in yeast. Yeast strain AH109 was transformed with indicated bait and prey fusion constructs. Overnight cultures of yeast transformants were dropped onto Complete Supplement Medium plates either lacking leucine and tryptophan (LW) or lacking leucine, tryptophan and adenin (LWAde) and incubated at $30^{\circ} \mathrm{C}$. Growth on LWAde medium indicates interaction between bait and prey fusion proteins. -ve denotes empty prey and bait plasmid. Photos were taken 2 days (LW) and 7 days (LWAde), respectively, after dropping. B) Quantification of FLIM analysis confirms direct protein-protein interaction between RIC157 and activated RACB in planta. GFP lifetime in barley epidermal cells transiently co-expressing indicated constructs was investigated at the aequatorial plane $2 \mathrm{~d}$ after transformation via particle bombardment. Graph shows result of 3 independent biological replicates. ${ }^{* * *}$ indicates significance $\mathrm{P}<10^{-4}$, Student's t-test. C) BiFC shows close proximity between RIC157 and RACB in planta. Barley epidermal 
1067 cells transiently co-expressing split-YFP fusion protein combinations and mCherry as 1068 cytosolic transformation marker after particle bombardment transformation. 2d after 1069 transformation, YFP fluorescence reconstitution was analysed via Confocal laser 1070 scanning microscopy. Shown are maximum projections of at least 15 optical sections 1071 taken at $2 \mu \mathrm{m}$ increments. Bars $=50 \mu \mathrm{m}$. 

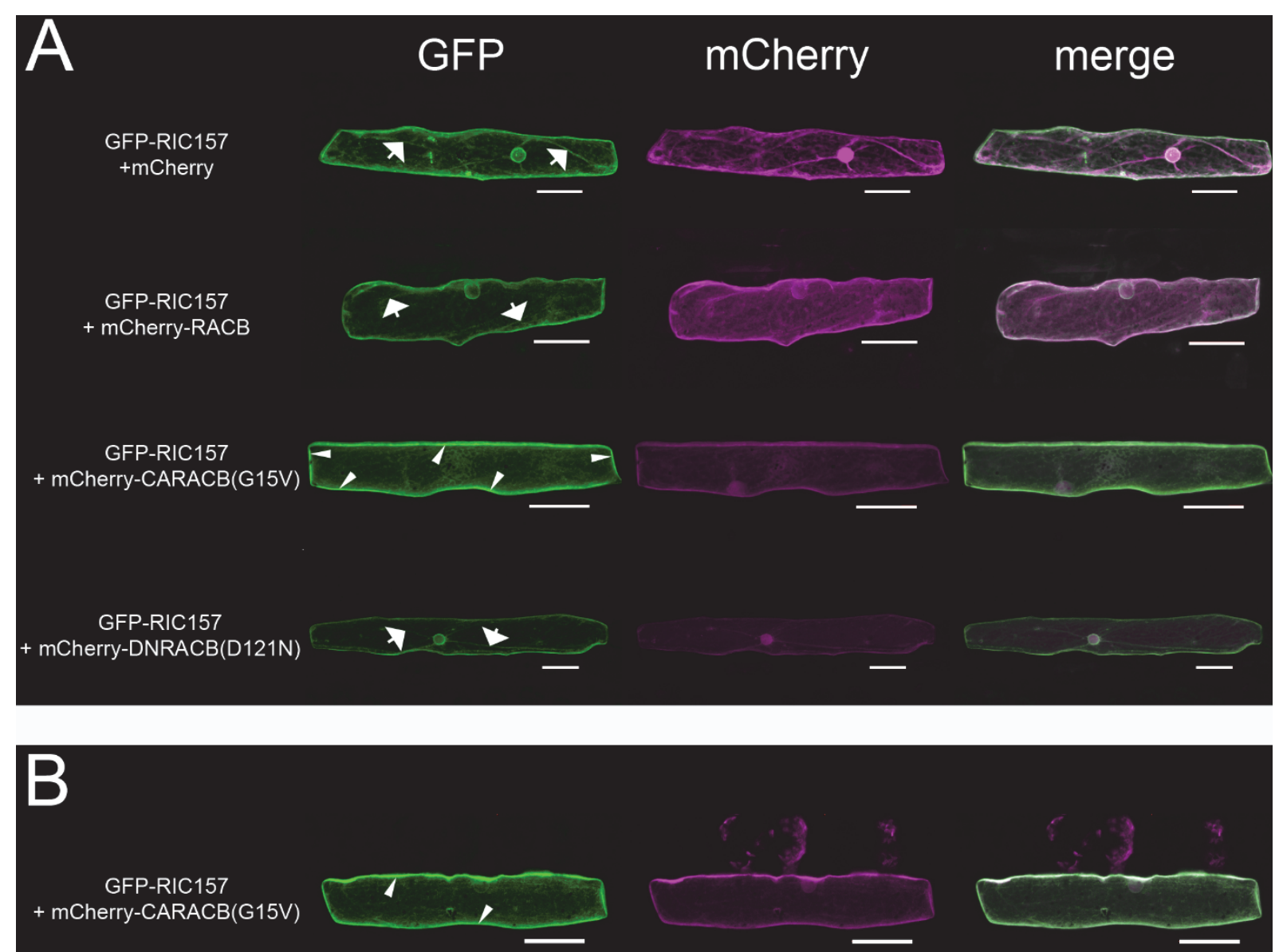

GFP-RIC157

+ mCherry-CARACB $(\mathrm{G} 15 \mathrm{~V})$ + RACB RNAi

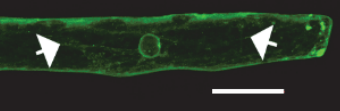

Figure 4: RIC157 localisation is affected by RACB-activation status. Confocal scanning microscopy of barley epidermal cells $1 \mathrm{~d}$ after transformation via particle bombardment. A) GFP-RIC157 localises to the cytoplasm, but not the nucleus (upper row) and is recruited to the cell periphery exclusively by mCherry-CARACB(G15V), but not by mCherry-RACB or mCherry-DNRACB(D121N).

B) Simultaneous RNA interference-mediated silencing of RACB attenuates RIC157 recruitment to the cell periphery. Arrows indicate cytoplasmic strands, arrow heads point towards GFP fluorescence accumulation at cell periphery. Microscopy pictures show maximum projections of at least 15 optical sections taken at $2 \mu \mathrm{m}$ increments. Bar $=50 \mu \mathrm{m}$. 


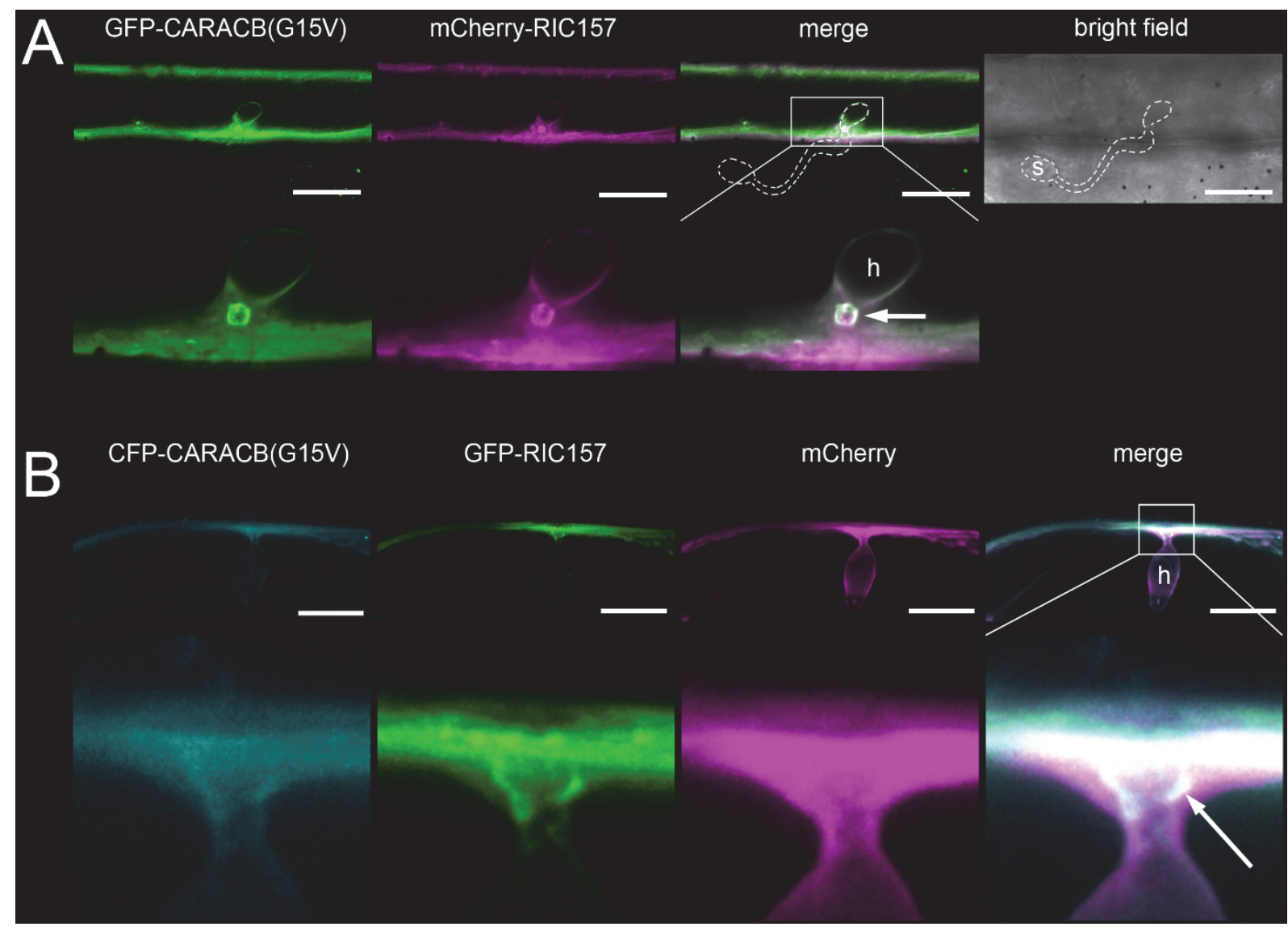

Figure 5: RIC157 is recruited to penetration site where it colocalises with activated RACB. Confocal laser scanning microscopy of epidermal cells 1d after transformation via particle bombardment and 18-24h after inoculation with Bgh. Cells in $A$ ) and $B$ ) show successful fungal penetration due to haustorium formation (h). A) Transient co-expression of GFP-CARACB(G15V) and mCherry-RIC157. Area in white square is enlarged in lower panel. $\mathrm{S}=$ spore; Bar $=30 \mu \mathrm{m} . \mathrm{B}$ ) Transient co-expression of CFP-CARACB(G15V) and GFP-RIC157. Cytosolic mCherry was expressed to distinguish RIC157 and CARACB(G15V) localisation from cytoplasm at penetration site. Bar $=20 \mu \mathrm{m}$. Arrows indicate approximate position of the haustorial neck. Contrast of images was equally slightly enhanced. Arrows indicate haustorial neck close to fungal penetration site. 

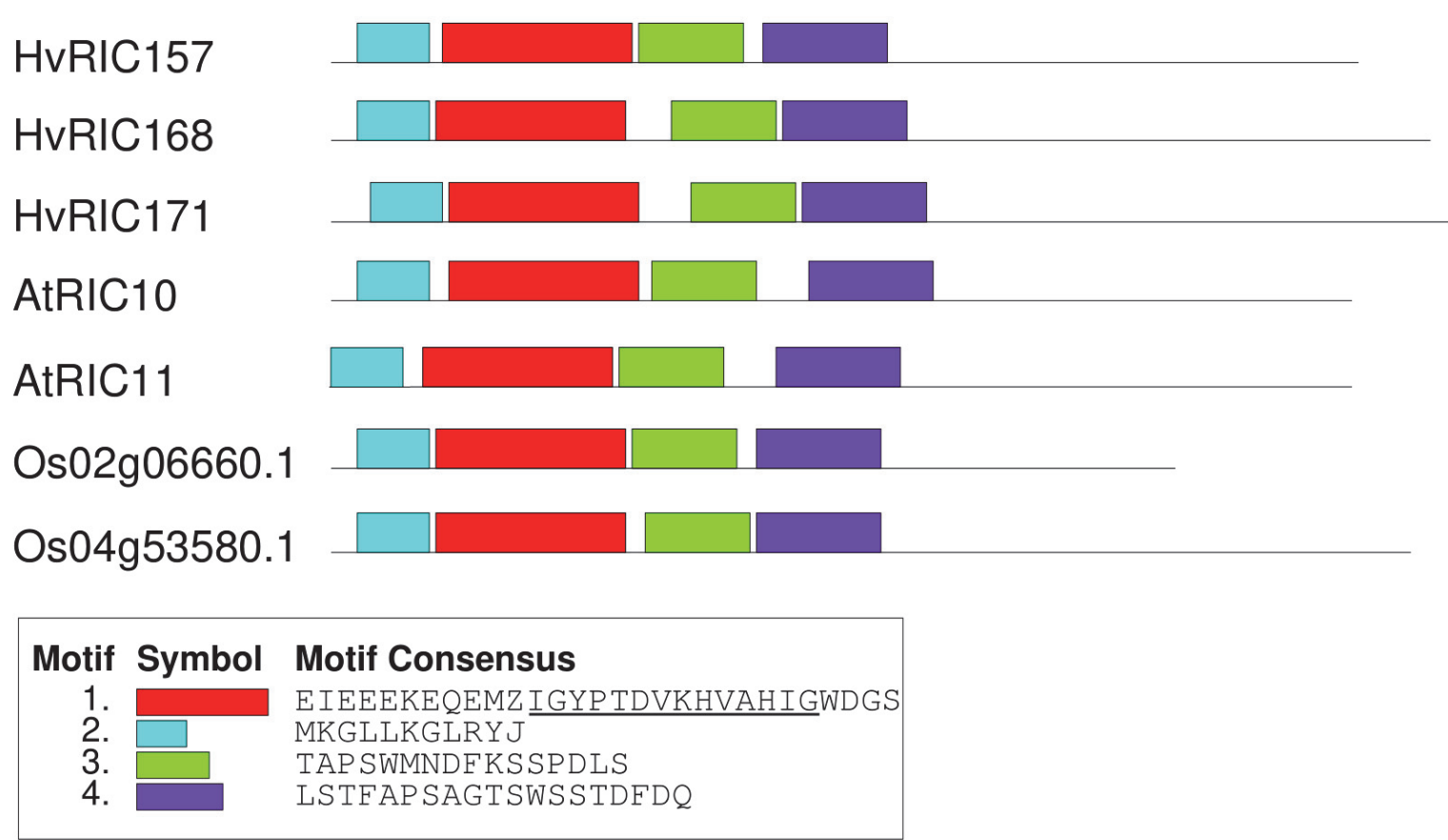

1100 Suppl. Fig. S1: Barley RIC157 shows limited sequence similarity to barley RIC168 and RIC171, but also to RIC proteins from Arabidopsis thaliana and Oryza sativa. Primary sequences of RIC proteins (schematically shown in top part of the figure) were analysed for domain homologies using MEME online software (http://memesuite.org/tools/meme). Consensus sequence of the four most similar motifs are shown below. Underlined sequence denotes CRIB domain. Hv = Hordeum vulgare, At = Arabidopsis thaliana, Os = Oryza sativa. 

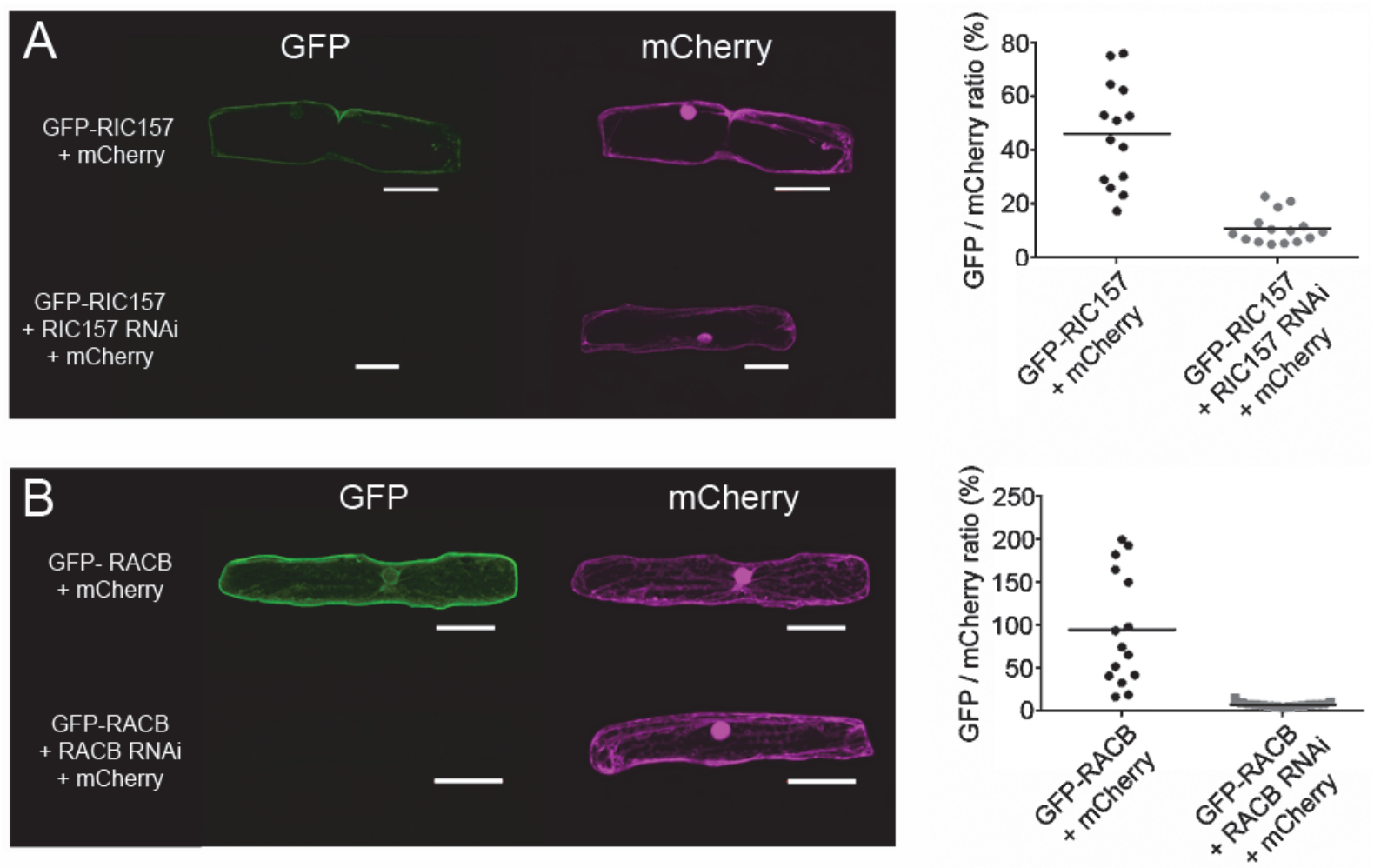

Suppl. Fig. S2: RNA interference-mediated silencing efficiency. Epidermal cells of $7 \mathrm{~d}$ old barley primary leaves were transiently transformed via particle bombardment with overexpression constructs of GFP fusions of RIC157 (A) and RACB (B) alone or together with RNAi silencing constructs. A construct to express cytosolic mCherry was simultaneously co-delivered for transformation efficiency and fluorescence quantification purposes. Microscopy images are maximum projections of at least 15 optical sections taken at $2 \mu \mathrm{m}$ increments. Bars $=50 \mu \mathrm{m}$. Each graph shows the mean of GFP fluorescence as percentage of mCherry fluorescence per transformed cell

1117 (whole cell area was taken as region of interest for measuring fluorescence intensity).

1118 Dots represent single measured cells. 


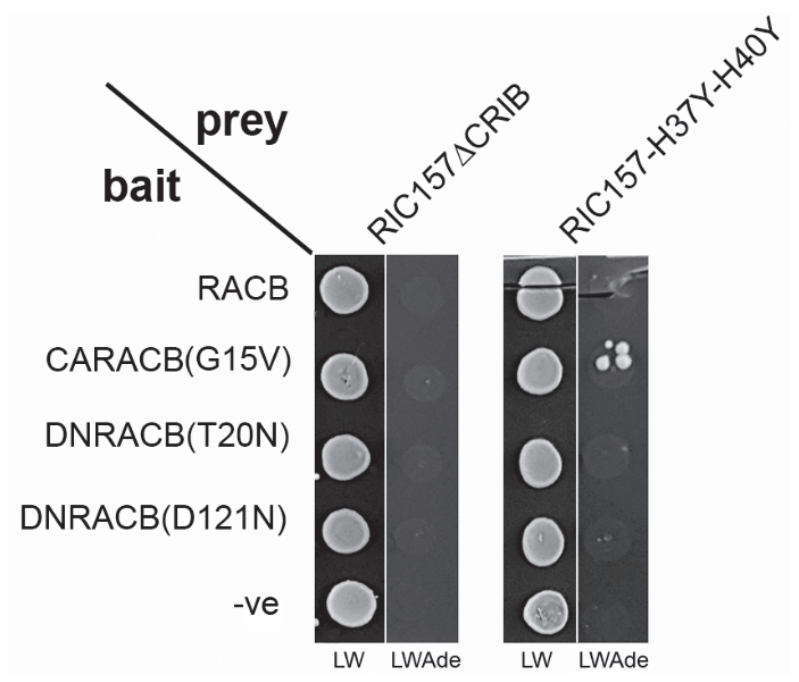

1121 Suppl. Fig. S3: CRIB deletion and CRIB mutation in RIC157 prevents interaction

1122 with RACB in yeast. Yeast strain AH109 was transformed with indicated bait and prey

1123 fusion constructs. Overnight cultures of yeast transformants were dropped onto

1124 Complete Supplement Medium plates either lacking leucine and tryptophan (LW) or

1125 lacking leucine, tryptophan and adenin (LWAde) and incubated at $30^{\circ} \mathrm{C}$. Growth on

1126 LWAde medium indicates interaction between bait and prey fusion proteins. -ve

1127 denotes empty prey and bait plasmid. Photos were taken 2 days (LW) and 7 days

1128 (LWAde), respectively, after dropping. 

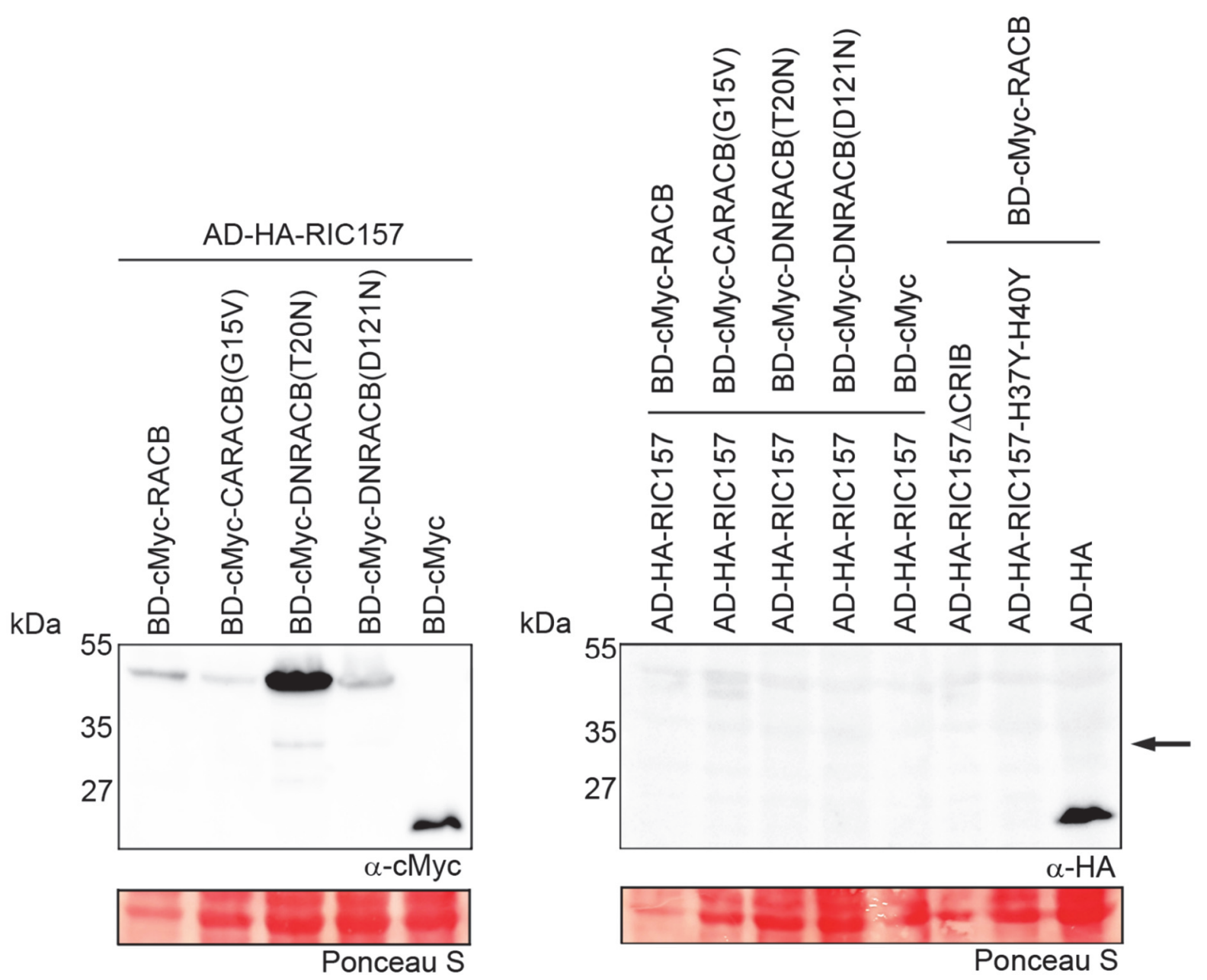

Suppl. Fig. S4: Protein stability in yeast. Immunoblots probed with $\alpha$-cMyc to detect RACB variants fused to GAL4 binding domain (BD; bait) encoded on pGBKT7 and $\alpha$ HA to detect RIC157 variants fused to GAL4 activation domain (AD; prey) encoded on pGADT7 after transformation of yeast strain AH109. Total yeast protein was extracted as described in Experimental Procedures. Molecular weight is indicated in kiloDalton (kDA). Ponceau S staining shows protein loading. Stable expression of RIC157 variants fused to GAL4 activation domain could not be detected in immunoblots (arrow indicates expected bandsize). 
A
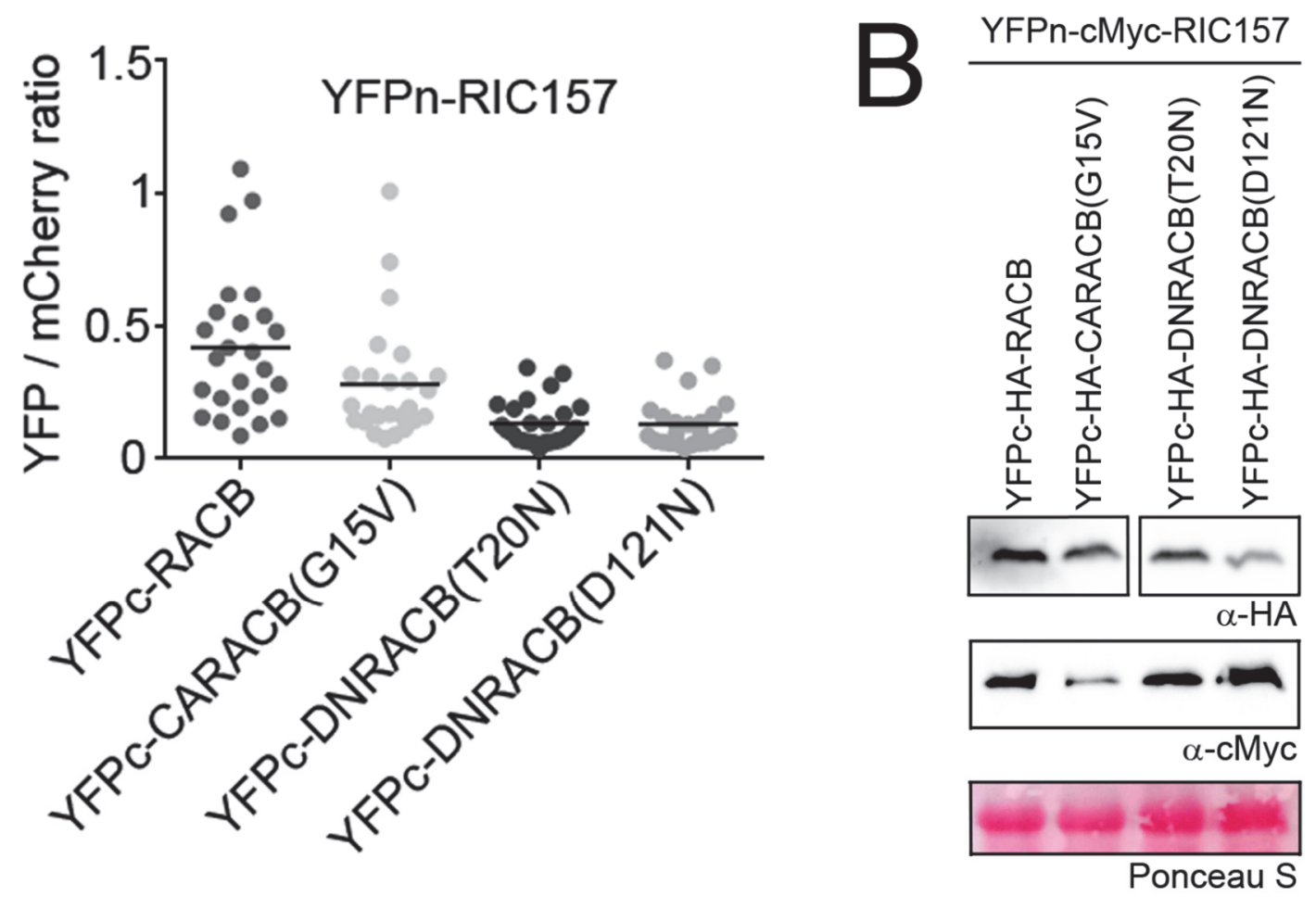

Suppl. Figure S5: Raciometric BiFC analyses and BiFC fusion protein stability.

A) Barley epidermal cells were transiently transformed with indicated constructs encoding for BiFC fusion proteins. Cytosolic mCherry expression was used in each sample as transformation marker and to quantify reconstituted YFP fluorescence ratiometrically. The whole cell area was taken as region of interest. Graph shows the mean of the YFP/mCherry fluorescence ratio, taken from at least 20 cells (shown as dots. B) Barley mesophyll protoplasts were prepared and transformed with BiFC constructs as described in Experimental procedures. To confirm stable in planta expression, immunoblots were probed with $\alpha-H A$ to detect YFPc-RACB fusion proteins and $\alpha$-cMyc to detect YFPn-RIC157 fusion proteins. Ponceau S staining shows equal protein loading. 


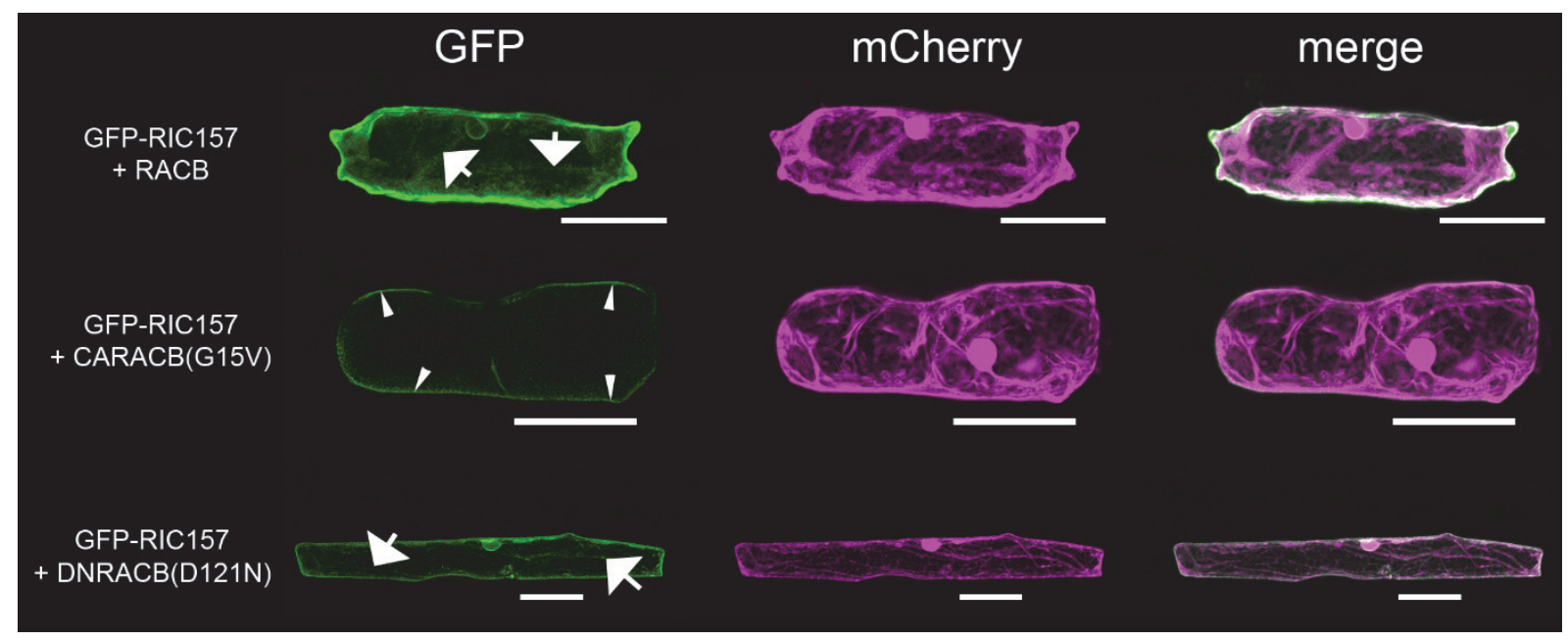

Suppl. Fig. S6: RIC157 is recruited to the cell periphery by activated RACB.

Confocal laser scanning microscopy of barley epidermal cells $1 \mathrm{~d}$ after transformation via particle bombardment. GFP-RIC157 localises to the cytoplasm, but not the nucleus and is recruited to the cell periphery exclusively by non-tagged CARACB(G15V), but not DNRACB(D121N). A construct for cytosolic mCherry expression was simultaneously used to check transformation efficiency. Arrows indicate cytoplasmic strands, arrow heads point towards GFP fluorescence accumulation at cell periphery. Microscopy pictures show maximum projections of at least 15 optical sections taken at $2 \mu \mathrm{m}$ increments. Bar $=50 \mu \mathrm{m}$.

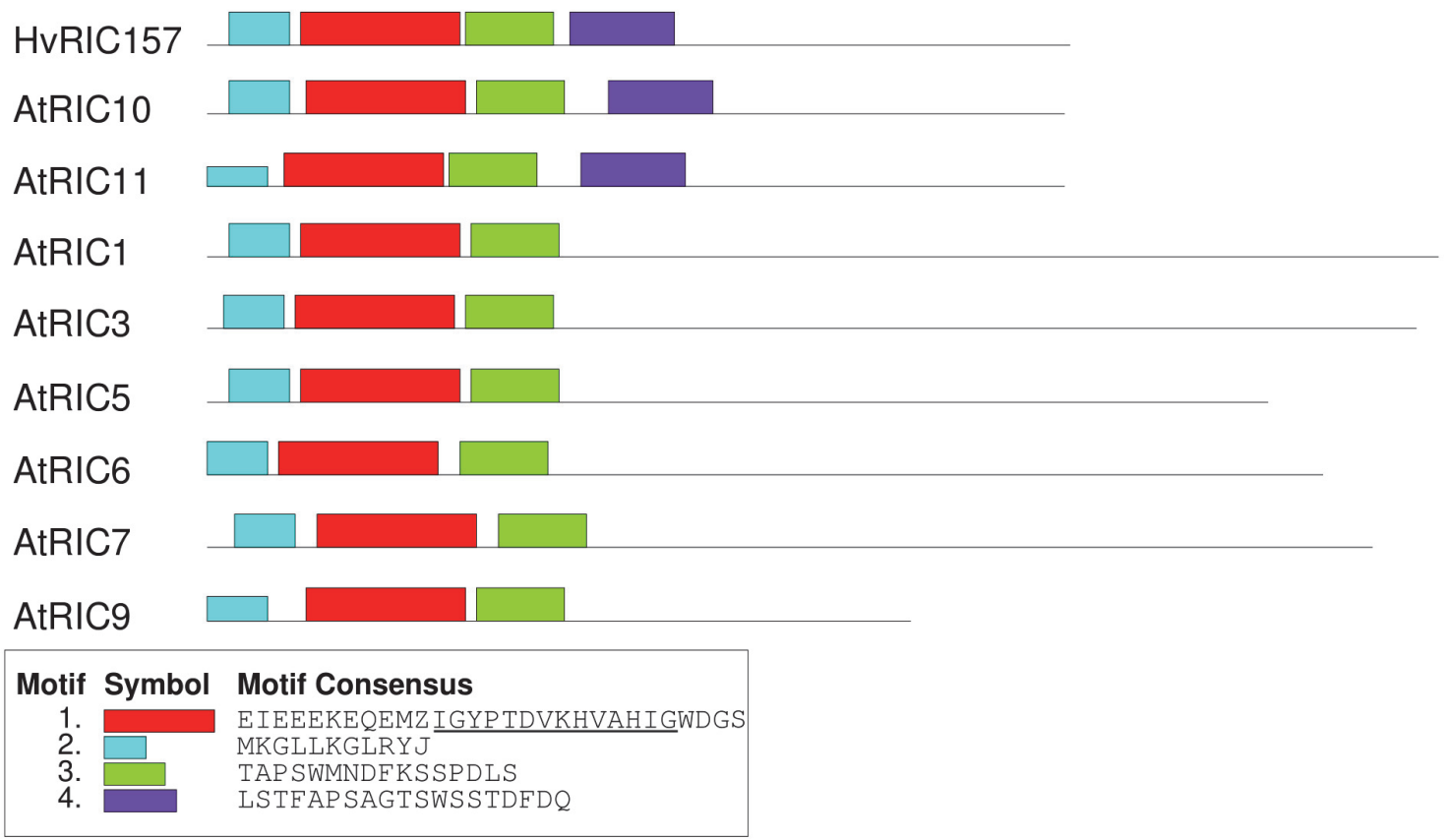




\begin{tabular}{|c|c|c|}
\hline Primer name & Gene (construct) & Sequence \\
\hline RIC157_GW for & $R / C 157$ & 5-AAAAAGCAGGCTCACAAATGGCGGTAAAGATGAAGGG-3* \\
\hline RIC157_GW rev+STOP & RIC157 & $5^{t}$-AGAAAGCTGGGTCACCGCCTCCGGATCAGACGACTCGAACCCCTCTTTGC-3i \\
\hline RIC157delCRIB for & RIC157 $\triangle C R I B$ & $5^{i}$-GCTCAAAAGGAGCATGAGATGGAATTGGGCACCAGTGACACATC- $3^{t}$ \\
\hline RIC157delCRIB rev & RIC157 $\triangle$ CRIB & $5^{2}$-CACTGGTGCCCAATTCCATCTCATGCTCCTTTTGAGC-3 ${ }^{2}$ \\
\hline CRIB157H37\&40Y for & RIC157-H37-40Y & 5-CCTACAGATGTAAAGTATGTGGCTTACATAGGTTTGGGCACCAGTGACACATCTCC-3' \\
\hline CRIB157H37\&40Y rev & RIC157-H37-40Y & 5'-GGAGATGTGTCACTGGTGCCCAAACCTATGTAAGCCACATACTTTACATCTGTAGG-3' \\
\hline attB1 & attB1 & 5'-GGGGACAAGTTTGTACAAAAAAGCAGGCTCACAA-3' \\
\hline attB2 & attB2 & 5'-GGGGACCACTTTGTACAAGAAAGCTGGGTCACCG-3* \\
\hline RIC157 RNAi_Notl for & RIC157 RNAi & 5'-AATTGCGGCCGCAAGATGAAGGGAATCTTCAAAGGGC $-3^{*}$ \\
\hline RIC157_RNAi_Xbal_rev & RIC157 RNAi & 5'-AATTTCTAGAACGCCGTCGCGAAGGAGGCCCTCGACC $-3^{t}$ \\
\hline RIC157 RNAi_EcoRI for & RIC157 RNAi & 5'-AATTGAATTCTTGGGCACCAGTGACACATCTCC- $3^{*}$ \\
\hline RIC157_RNAi_EcoRI rev & RIC157 RNAi & 5-AATTGAATTCTTCCATCTCATGCTCCTTTTGAGC-3* \\
\hline RIC157_BamHI for & $R / C 157$ & 5'-AATTGGATCCATGGCGGTAAAGATGAAGGGAATC- ${ }^{2}$ \\
\hline RIC157_Kpnl_rev & RIC157 & 5-AATTGGTACCCTAGACGACTCGAACCCCTCTTTGC-3' \\
\hline RIC157 for & RIC157 & $5^{4}$-ATGGCGGTAAAGATGAAGG-3t \\
\hline RIC157 rev & RIC157 & 5'-GACGACTCGAACCCCTCTTTGC-3 $3^{4}$ \\
\hline RACB D121N fw & DNRACB(D121N) & 5'-CTCGTGGGAACAAAGCTTAATCTTCGAGATGACAAG-3 ${ }^{\star}$ \\
\hline RACB D $121 \mathrm{~N}$ rv & DNRACB(D121N) & $5^{*}$-CTTGTCATCTCGAAGATTAAGCTTTGTTCCCACGAG-3' \\
\hline RACB_GW for & $R A C B$ & 5-AAAAAGCAGGCTCACAAATGAGCGCGTCCAGGTTCATAAAGTGC- ${ }^{2}$ \\
\hline RACB GW rev & RACB & 5'-AGAAAGCTGGGTCACCGCCTCCGGACAAGATGGAGCAAGCCCCCC-3 ${ }^{\star}$ \\
\hline delCSIL for & $R A C B \triangle C S I L$ & 5'-GAAGAAAAAGGCGCAGAGGGGGGCTTGATCCATCTTGTAGTCCGGAGGCGGTG-3' \\
\hline delCSIL rev & $R A C B \triangle C S I L$ & $5^{*}$-CACCGCCTCCGGACTACAAGATGGATCAAGCCCCCCTCTGCGCCTTTTTCTTC-3 ${ }^{*}$ \\
\hline GW RfA mCherry-F & mCherry & 5'-GCTGTACAAGATCACAAGTTTGTACAAAAAAGCTG-3i \\
\hline GW RfA meGFP-F & meGFP & $5^{2}$-GCTGTACAAAATCACAAGTTTGTACAAAAAAGCTG- ${ }^{2}$ \\
\hline GW RfA Xba-R & RfA & $5^{t}$-TGCCTGCAGGTCGACTCTAGAATCACCACTTTGTACAAGAAAGCTG-3 ${ }^{t}$ \\
\hline GW Xba_RfB-F & RfB & $5^{\prime}$-GGTACCCGGGGATCCTCTAGAATCAACAAGTTTGTACAAAAAAGCT-3 \\
\hline GW RfB-R & RfB & $5^{t}$-TGCTCACCATATCAACCACTTTGTACAAGAAAGCT- $3^{t}$ \\
\hline meGFP-STP-F & meGFP & $\begin{array}{l}5^{i} \text {-AGTGGTTGATATGGTGAGCAAGGGCGAGG-3 } \\
\end{array}$ \\
\hline mCherry-STP-F & mCherry & $5^{*}$-AGTGGTTGATATGGTGAGCAAGGGCGAGG-3 ${ }^{x}$ \\
\hline XFP-noSTP Xba-F & $X F P$ & $5^{2}$-GGTACCCGGGGATCCTCTAGAATGGTGAGCAAGGGCGAGG-3 ${ }^{2}$ \\
\hline XFP-noSTP-R & $X F P$ & 5'-AACTTGTGATCTTGTACAGCTCGTCCATGCC- $3^{i}$ \\
\hline meGFP-noSTP-R & meGFP & 5-AACTTGTGATTTTGTACAGCTCGTCCATGCC-3' \\
\hline mCherry-STP Xba-R & mCherry & $5^{\prime}$-TGCCTGCAGGTCGACTCTAGATTACTTGTACAGCTCGTCCATGCC- $3^{2}$ \\
\hline meGFP-STP Xba-R & meGFP & 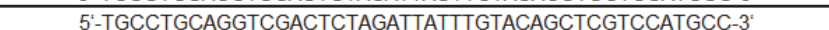 \\
\hline
\end{tabular}

\section{Suppl. Table 1: Primers used in this study}

